\title{
Chapter 14 \\ Resonance Energy Transfer: Theoretical Foundations and Developing Applications
}

\section{David L. Andrews}

University of East Anglia Norwich, Norwich, UK

\subsection{Introduction}

14.1.2 The nature of condensed phase energy transfer

14.1.3 The Förster equation

14.1.4 Established areas of application

14.2 Electromagnetic Origins

14.2.1 Coupling of transition dipoles

14.2.2 Quantum electrodynamics

14.2.3 Near- and far-field behaviour

14.2.4 Refractive and dissipative effects

14.3 Features of the Pair Transfer Rate

14.3.1 Distance dependence

14.3.2 Orientation of the transition dipoles

14.3.3 Spectral overlap

14.4 Energy Transfer in Heterogeneous Solids

14.4.1 Doped solids

14.4.2 Quantum dots

14.4.3 Multichromophore complexes

14.5 Directed Energy Transfer

14.5.1 Spectroscopic gradient

14.5.2 Influence of a static electric field

14.5.3 Optically controlled energy transfer

14.6 Developing Applications

14.7 Conclusion

References 


\subsection{Introduction}

In the wide range of materials that are characterised by broad, relatively featureless optical spectra, the absorption of light in the ultraviolet-visible wavelength region is typically followed by rapid internal processes of dissipation and degradation of the acquired energy, the latter ultimately to be manifest in the form of heat. In more complex materials-those comprising a variety of lightabsorbing atomic or molecular components (chromophores) with optically well characterised absorption and fluorescence bands-the absorption of light is commonly followed by a spatial translation of the absorbed electromagnetic radiation between different, though usually closely separated, chromophores. The process takes place well before the completion of any thermal degradation in such materials. This primary relocation of the acquired electronic energy, immediately following photo-excitation, is accomplished by a mechanism that has become known as resonance energy transfer (RET). ${ }^{1-3}$ (At an earlier stage in the development of these ideas, ${ }^{4}$ the term 'resonance' was used to signify that no molecular vibrations were excited; however, such usage is now known to be relevant to few systems and has largely fallen into abeyance.) An alternative designation for the process is electronic energy transfer (EET); both terms are widely used, and in each case, the first letter of the acronym serves as a distinction from electron transfer.

In complex multichromophore materials, the singular properties of RET allow the flow of energy to exhibit a directed character. Because the process operates most efficiently between near-neighbor chromophores, the resonance propagation of energy through such a system generally takes the form of a series of short steps; an alternative process involving fewer long steps proves considerably less favorable. In suitably designed materials, the pattern of energy flow following optical absorption is thus determined by a sequence of transfer steps, beginning and ending at chromophores that differ chemically, or, if the chromophores are structurally equivalent, through local modifications in energy level structure reflecting the influence of their electronic environment. Hence. individual chromophores that act in the capacity of excitation acceptors can subsequently adopt the role of donors. This effect contributes to a crucial, property-determining characteristic for the channeling of electronic excitation in photosynthetic systems; ${ }^{5}$ the same principles are emulated in synthetic energy harvesting systems such as the fractal polymers known as dendrimers. ${ }^{6}$

The observation and applications of RET extend well beyond the technology of light harvesting, as will be demonstrated in later sections of this chapter. The phenomenon has an important function in the operation of organic light-emitting diodes (OLEDs) and luminescence detectors; in crystalline solids and glasses doped with transition metal ions, mechanisms based on RET are also engaged for laser frequency conversion. In the fields of optical communications and computation, several optical switching and logic gate devices are founded on the same principle. As we shall see, those possibilities have been considerably extended by a recent discovery that electron spin can be transferred along with the energy. In the realm of molecular biology, the determination of protein 
structures and the characterisation of dynamical processes is furthered by studies of the transfer of energy between intrinsic or 'tag' chromophores; other ultrasensitive molecular imaging applications are again based on the same underlying principle. Further applications include energy transfer systems designed to act as analyte-specific sensors, and as sensitisers for photodynamic therapy. Last but not least, RET provides a rich ground for exploring the fundamental issues that arise from the nanoscale interplay of electromagnetism and quantum mechanics.

\subsubsection{The nature of condensed phase energy transfer}

In any nonhomogeneous dielectric material, the primary result of ultraviolet/visible absorption is the population of short-lived electronic excited states located in individual atomic/molecular/nanoscale centres. In general, this is immediately followed by one or more transfers of the acquired electronic excitation energy, commonly on an ultrafast timescale and with some associated losses such as vibrational dissipation. Given the broad compass of the term 'condensed phase,' it is really quite astonishing that the RET concept is so pervasive, and the fundamental theory so extensively applicable. In crystalline, semicrystalline, or glassy media, the centres of absorption (and subsequent reemission) commonly take the form of ions, atoms, or colour centres; in other types of mediums they may be small molecules, electronically distinct parts of large molecules, or nanoparticles such as quantum dots. Where generality is intended in the following, the term 'chromophore' is used, subsuming the term 'fluorophore,' which some employ to convey the frequently associated capacity to exhibit fluorescence. Although the energy flow that follows optical absorption is generally a multistep process, at the fundamental level, each elementary transfer step is a radiationless pairwise interaction, generally taking place between an electronically excited species termed the donor and an electronically distinct acceptor that is initially in its ground state. The theory is therefore based on pair couplings.

The primary equations for pair RET are based on the interactions of nonoverlapping transition dipoles. Before pursuing the detail, however, it is worth observing that other forms of coupling are also possible, though less relevant to most systems of interest in the following account. For example, the transfer of energy between particles or units with significantly overlapped wavefunctions is usually described in terms of Dexter theory, ${ }^{7}$ where the coupling carries an exponential decay with distance, directly reflecting the radial form of the wavefunctions and electron distributions. Compared to materials in which the donor and acceptor orbitals do not spatially overlap, such systems are of less use for either device or analytical applications, largely because the coupled chromophores lose their electronic and optical integrity. This is the main reason that complex light-harvesting systems are commonly designed with nonconjugated linkages or spacer units between the chromophores, or else with the latter held on a host superstructure that prevents direct chromophore contact. 
Parallels can be drawn with the way a dielectric layer operates in a simple electrical capacitor. In the alternative scenario, where spacer units act as a 'conductive' bridge through delocalisation and mixing of their orbitals with the donor or acceptor orbitals, energy transfer is specifically expedited by the operation of a superexchange mechanism, ${ }^{8,9}$ which, despite the efficiency gains, compromises diagnostic applications.

\subsubsection{The Förster equation}

The first theoretical formulation of pair transfer that successfully identified the inverse sixth power distance dependence (with which the process itself is now almost universally associated) was made by Förster, ${ }^{10}$ and experimentally verified by Latt et al. ${ }^{11}$ Subsequently recast in quantum mechanical terms, this theory of 'radiationless' energy transfer has been very successfully applied for well over half a century and remains widely valid, although subject to certain conditions that were not originally understood. Before proceeding with the detail of the Förster equation, a caveat is therefore due. This concerns a misunderstanding of the relationship between 'radiationless' and 'radiative' energy transfer (the latter signifying successive but distinct processes of fluorescence emission by the donor, and capture of the ensuing photon by the acceptor). A full quantum electrodynamical treatment of the interaction to was needed to clarify and resolve this issue (details are given in Section 14.2.2). However, the reader should be aware that some textbooks still obscure the subject, wrongly treating radiationless and radiative energy transfer between a given chromophore pair as separate, potentially competing processes.

To proceed, consider the pairwise transfer of excitation between two chromophores $A$ and $B$. In the context of this elementary mechanism (which might be one RET component of a complex, multistep migration process), $A$ is designated the donor and $B$ the acceptor. Specifically, let it be assumed that prior excitation of the donor generates an electronically excited species $A^{*}$. Forward progress of the energy is then accompanied by donor decay to the ground electronic state. Acquiring the energy, the acceptor $B$ undergoes a transition from its ground to its excited state, as illustrated in Fig. 14.1. The excited acceptor $B^{*}$ subsequently decays either in a further transfer event, or by another means such as fluorescence.

Because the $A^{*}$ and $B^{*}$ states are real, having measurable lifetimes, the process of energy transfer itself is fundamentally separable from the initial electronic excitation of $A$ and the eventual decay of $B$; the latter processes do not, therefore, enter into the theory of the pair transfer. However it will need to be registered that other dissipative processes may be engaged (such features will be discussed in detail in later sections). In a solid, the linewidth of optical transitions manifests a degree of coupling of individual optical centres with their electronic environment (which, in the case of strong coupling, may lead to the production of phonon side-bands). Similar effects in solutions or disordered solids represent 


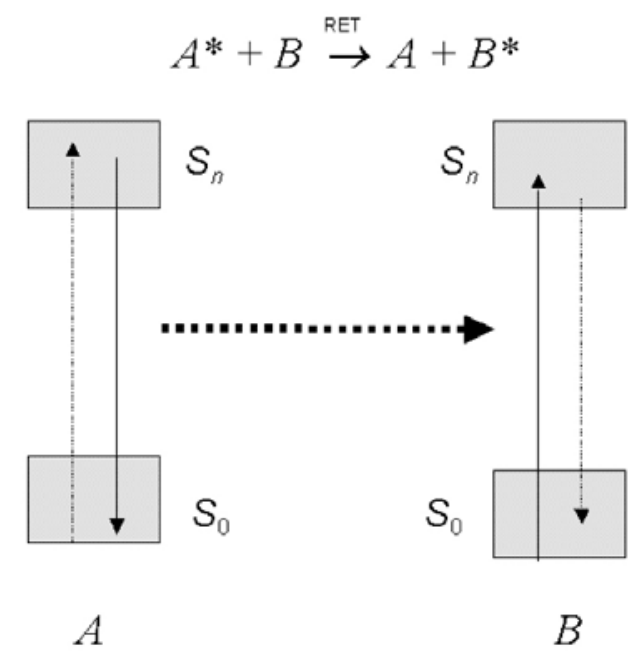

Figure 14.1 Energetics of single-step resonance energy transfer, also showing the preceding excitation of the donor $A$ and the concluding decay of the acceptor $B$. The boxes indicate lower (ground) and excited electronic states (here, designated as singlet states, though they need not be), and the vertical width represents a finite breadth on the energy scale. The transferred energy may be less than that initially acquired by the donor, due to dissipative processes; a similar remark applies to the acceptor.

inhomogeneous interactions with a solvent or host, while the broad bands exhibited by chromophores in complex molecular systems signify extensively overlapped vibrational levels, including those associated with skeletal modes of the superstructure. In each case, the net effect is to allow pair transfer to occur at any energy level within the region of overlap between the donor emission and acceptor absorption bands.

Restricting consideration to donor-acceptor separations $R$ substantially smaller than the wavelengths of visible radiation, the Förster theory gives the following expression for the rate of pairwise energy transfer $w_{\mathrm{F}}$, for systems where the host material for the donor and acceptor has refractive index $n$ (at the optical frequency corresponding to the mean transferred energy):

$$
w_{\mathrm{F}}=\frac{9 \kappa^{2} c^{4}}{8 \pi \tau_{A^{*}} n^{4} R^{6}} \int F_{A}(\omega) \sigma_{B}(\omega) \frac{d \omega}{\omega^{4}} .
$$

In this expression, $F_{A}(\omega)$ is the fluorescence spectrum of the donor (normalised to unity); $\tau_{A^{*}}$ is the associated radiative decay lifetime (related to the measured fluorescence lifetime $\tau_{\mathrm{fl}}$ through the fluorescence quantum yield $\left.\eta=\tau_{\mathrm{fl}} / \tau_{\mathrm{A}^{*}}\right) ; \sigma_{\mathrm{B}}(\omega)$ is the linear absorption cross-section of the acceptor; $\omega$ is an optical frequency in radians per second; and $c$ is the speed of light. The spectral functions $F_{A}$ and $\sigma_{B}$ are mathematically defined and discussed in detail in Section 14.2.1. The $\kappa$ factor in Eq. (14.1) depends on the orientations of the donor and 
acceptor, both with respect to each other, and with respect to their mutual displacement unit vector $\hat{\mathbf{R}}$, as follows:

$$
\kappa=\left(\hat{\boldsymbol{\mu}}_{A} \cdot \hat{\boldsymbol{\mu}}_{B}\right)-3\left(\hat{\mathbf{R}} \cdot \hat{\boldsymbol{\mu}}_{A}\right)\left(\hat{\mathbf{R}} \cdot \hat{\boldsymbol{\mu}}_{B}\right)
$$

For each chromophore, $\hat{\boldsymbol{\mu}}$ designates a unit vector in the direction of the appropriate transition dipole moment (see Section 14.2.1). Unfavorable orientations can reduce the rate of energy transfer to zero; others, including many of those found in nature, optimise the transfer rate. The angular disposition of chromophores is therefore a very important facet of energy transfer, and one that invites careful consideration in the design of light-harvesting materials. To correct a common misconception, note that transfer is not necessarily precluded when the transition moments lie in perpendicular directions, provided that neither is orthogonal to $\mathbf{R}$ (=R $\hat{\mathbf{R}}$ ). The most striking features exhibited by Eq. (14.1) are the dependences on distance and on spectral overlap, both of which will be examined in detail in following sections.

In experimental studies of RET, it is usually significant that the electronically excited donor can in principle release its energy by spontaneous decay, and that the ensuing fluorescence radiation is detectable by any suitably placed photodetector. Because the alternative possibility (that of energy being transferred to another chromophore within the system) has such a sharp decline in efficiency as the distance to the acceptor increases, it is commonplace to introduce the concept of a critical distance $R_{0}$, a separation at which the theoretical rates of RET and spontaneous emission by the donor are equal (now known as the Förster distance). The Förster rate equation is often cast in an alternative form, exactly equivalent to Eq. (14.1), explicitly exhibiting this critical distance: ${ }^{12}$

$$
w_{\mathrm{F}}=\frac{3 \kappa^{2}}{2} \frac{1}{\tau_{A^{*}}}\left(\frac{\bar{R}_{0}}{R}\right)^{6}
$$

Here, $\bar{R}_{0}$ is defined as the Förster distance for which the orientation factor $\kappa^{2}$ assumes its isotropic average value, $2 / 3 \cdot{ }^{13}$ For complex systems, the angular dependence is quite commonly disregarded and the following simpler expression employed:

$$
w_{\mathrm{F}}=\frac{1}{\tau_{A^{*}}}\left(\frac{R_{0}}{R}\right)^{6}
$$

leading to a transfer efficiency $\Phi_{\mathrm{T}}$ expressible as: 


$$
\Phi_{\mathrm{T}}=\frac{1}{1+\left(R / R_{0}\right)^{6}} .
$$

Typical values of the Förster radius range over a few nanometers. Thus, when a given electronically excited chromophore is within a distance $R_{0}$ of a suitable acceptor, RET will generally be the dominant decay mechanism; conversely, for distances beyond $R_{0}$, spontaneous decay will be the primary means of donor deactivation.

\subsubsection{Established areas of application}

In a host of multichromophore materials, RET represents a mechanism whose operation exerts a major influence on optical properties. Wide-ranging as these systems and applications are (several solid state device-oriented applications are to be discussed in later sections), the most widely studied and characterised examples are found in connection with light harvesting-both biological and synthetic. Following the capture of a photon by any such system, the RET mechanism dictates that the migration of the acquired energy from the site of the initial photoabsorption through to the site of its utilisation is at every stage subject to an inverse sixth-power dependence on distance. As a result, energy migration over distances beyond the Förster radius primarily operates through a series of short hops rather than one long hop. Commonly, these hops exhibit a 'spectroscopic gradient, ${ }^{14}$ a term for the progressively longer wavelengths for absorption and fluorescence in successively visited chromophores (a feature to be studied in detail in Section 14.5.1). This is a property that makes a significant contribution to the high efficiency of photosynthetic and allied systems.

In experimental science, one obvious application of the strong distance dependence is the identification of motions in molecules, or parts of molecules, that can bring one chromophore into the proximity of another; prominent examples in biology are the traffic across a cell membrane, and protein folding. ${ }^{15}$ These and other such processes can be registered by selectively exciting one chromophore using laser light, and monitoring either the decrease in fluorescence from that species, or the rise in (again, generally longer-wavelength) fluorescence from the other chromophore as it enters into the role of acceptor. The judicious use of optical dichroic filters can make this fluorescence RET or 'FRET' technique perfectly straightforward (see Fig. 14.2). In cases where the two material components of interest do not display both absorption and fluorescence features in an appropriate wavelength range, molecular tagging with site-specific 'extrinsic' (i.e., artificially attached) chromophores can solve the problem.

In some applications, the actual distance between the chromophore groups is of specific interest. When the same two chromophores feature (in spatially different configurations) in the chemical composition of two different systems (again, a common feature in biology), the relative displacements of the chromophores can be quantitatively assessed on the basis of comparisons 


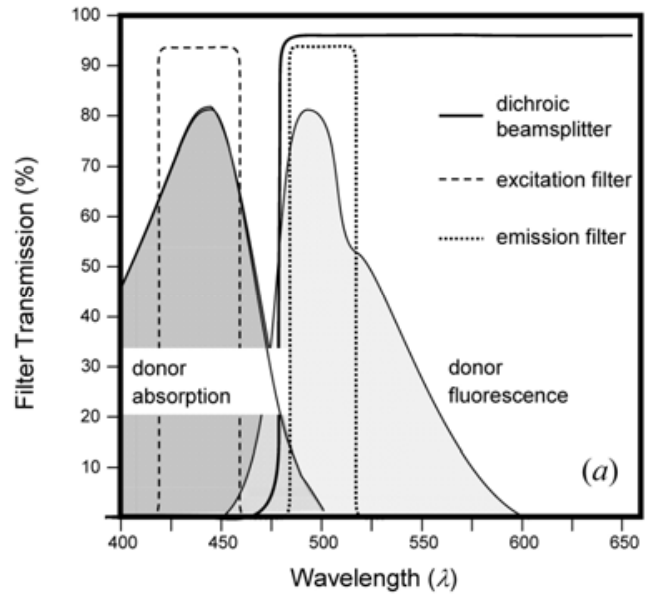

(a)

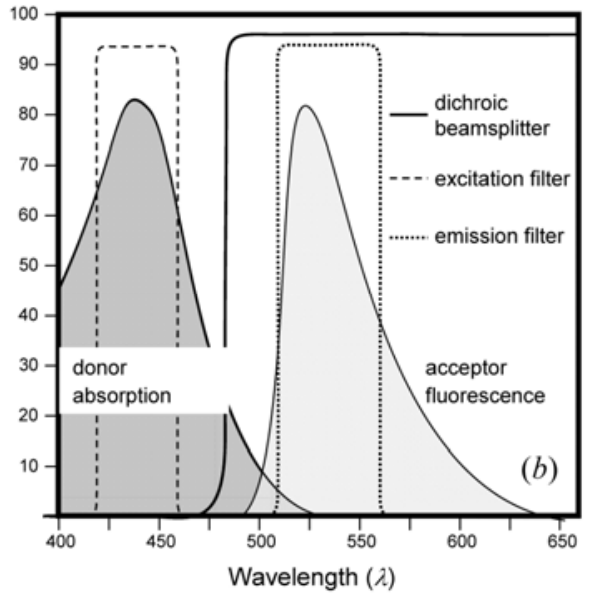

(b)

Figure 14.2 Schematic depiction of the spectral resolution of fluorescence from donor and acceptor species (based on cyan fluorescent protein donor and yellow fluorescent protein acceptor). (a) The transmission curve of a short-wavelength filter ensures initial excitation of the donor; a dichroic beam splitter and narrow emission filter ensure that only the (Stokes-shifted) fluorescence from the donor reaches a detector. (b) In the same system, a longer-wavelength emission filter ensures capture of only the acceptor fluorescence, following RET.

between the corresponding RET efficiencies. Such a technique is popularly known as a 'spectroscopic ruler.' ${ }^{16}$. Such elucidations of molecular structure usually lack information on the relative orientations of the groups involved, and as such, the calculations usually ignore the kappa parameter [Eq. (14.2)]. The apparent crudeness of this approach becomes more defensible on realising that even if it were to introduce a factor of two inaccuracy, the deduced group spacing would still be in error by only $12 \%$ (since $2^{1 / 6}=1.12$ ).

\subsection{Electromagnetic Origins}

In this section, the detailed electromagnetic origins of RET coupling are considered. First it is shown how the Förster formula signifies transition dipole coupling, and the possible influence of other multipoles is considered. An outline is then given of the modern and fully rigorous quantum electrodynamical derivation. Some distinctive features of the latter-in particular, the short- and long-range behaviour - are then examined in detail.

\subsubsection{Coupling of transition dipoles}

The spectral functions that were featured in Eq. (14.1) can be written in terms of the fundamental quantum properties of the chromophores. Assuming the usual Born-Oppenheimer separation of electronic and nuclear motions, the mathematical definitions are expressible as follows, using Dirac notation: 


$$
\begin{aligned}
& F_{A}(\omega)=\frac{\omega^{3} \tau_{A^{*}} \mu_{A}^{2}}{3 \varepsilon_{0} \pi c^{3}} \sum_{n, r} \rho_{A^{*}}^{(n)}\left|\left\langle\varphi_{A}^{(r)} \mid \varphi_{A^{*}}^{(n)}\right\rangle\right|^{2} \delta\left(E_{A_{n}^{*}}-E_{A_{r}}-\hbar \omega\right) ; \\
& \sigma_{B}(\omega)=\frac{\pi \omega \mu_{B}^{2}}{3 \varepsilon_{0} c} \sum_{m, p} \rho_{B}^{(m)}\left|\left\langle\varphi_{B^{*}}^{(p)} \mid \varphi_{B}^{(m)}\right\rangle\right|^{2} \delta\left(E_{B_{p}^{*}}-E_{B_{m}}-\hbar \omega\right) .
\end{aligned}
$$

Here, $\mu_{A}$ and $\mu_{B}$ are the magnitudes of the transition electric dipole moments for the donor decay and acceptor excitation, specifically given by:

$$
\boldsymbol{\mu}_{A}=\left\langle\psi_{A}|\boldsymbol{\mu}| \psi_{A^{*}}\right\rangle ; \boldsymbol{\mu}_{B}=\left\langle\psi_{B^{*}}|\boldsymbol{\mu}| \psi_{B}\right\rangle,
$$

where the $\mu$ is the dipole operator and each $\psi$ is an electronic state wavefunction. Furthermore, the indices $m, n, p$, and $r$ in Eq. (14.7) are generic labels denoting vibrational sublevels, with each $\varphi$ representing an associated wavefunction and $E$ the corresponding energy, and $\rho$ denoting a population distribution function for the initial state of each species. Comparing the above results with Eqs. (14.1) and (14.2) reveals the intrinsic quadratic dependence of the energy transfer rate on a coupling of the form

$$
C=\frac{\boldsymbol{\mu}_{A} \boldsymbol{\mu}_{B}}{4 \pi \varepsilon_{0} R^{3}}\left[\left(\hat{\boldsymbol{\mu}}_{A} \cdot \hat{\boldsymbol{\mu}}_{B}\right)-3\left(\hat{\mathbf{R}} \cdot \hat{\boldsymbol{\mu}}_{A}\right)\left(\hat{\mathbf{R}} \cdot \hat{\boldsymbol{\mu}}_{B}\right)\right],
$$

which is, of course, the usual formula for the interaction of two static dipoles. However, the result given by Eq. (14.9) is not a conventional energy of interaction, but a quantum amplitude (strictly speaking, it is an off-diagonal matrix element connecting different initial and final states; only diagonal terms can directly signify energy). This is one of several important distinctions, the significance of which will shortly become more apparent when the quantum electrodynamical theory is developed.

It is evident from Eq. (14.9), that the familiar inverse sixth-power distance dependence of RET owes its origin to the quadratic dependence of its rate on a coupling of transition electric dipoles. The result is, of course, applicable only when both the donor decay and the acceptor excitation transitions are electric dipole (E1) allowed. In general, the coupling is effected by a coupling between the lowest orders of multipoles (electric, or magnetic) that can support the necessary transition. In the Förster range, the distance dependence exhibits the form $R^{-(P+Q+1)}$ for the coupling of two transition electric multipoles $E P-E Q$, or two magnetic multipoles $\mathrm{MP}-\mathrm{MQ}$; while for the coupling of an electric multipole with a magnetic multipole, $\mathrm{E} P-\mathrm{MQ}$, the distance dependence is $R^{-(P+Q)} \cdot{ }^{17,18}$ For example, the coupling of an electric dipole decay with an electric quadrupole excitation, E1-E2, has an $R^{-4}$ distance dependence within the Förster range. However, it should be kept in mind that each unit increase in multipolar order, 
and each substitution of an electric transition by a magnetic counterpart, lowers the strength of the coupling by a factor of the order of $10^{-2}$ to $10^{-3}$. The decreasing efficiency of successive multipole orders increasingly disfavors the role of RET in the decay of the donor, compared to other decay mechanisms.

\subsubsection{Quantum electrodynamics}

To develop a theory of RET from a fully rigorous basis, it makes sense to use a foundation in which quantum mechanics is used to describe the behaviour of both the matter and any electromagnetic fields that are involved. This is the framework of quantum electrodynamics (QED). ${ }^{19,20}$ This theory has the advantage that it naturally accommodates not only quantum mechanical but also relativistic principles, so that it properly delivers retarded solutions (which will shortly prove to be a matter of key significance for RET). The wider successes of QED, such as its prediction of the Casimir effect, are well known; less well known is the fact that it alone can rigorously explain the much more familiar process of spontaneous emission. Moreover, a good case can be made that even the use of electric and magnetic multipoles is only defensible in the context of a fully quantum electrodynamical theory. ${ }^{21}$ The development of a QED theory of RET, which began over forty years ago with pioneering work by Avery, Gomberoff, and Power, ${ }^{22,23}$ culminated twenty years later in a unified theory ${ }^{24}$ that has ramifications that continue to be explored today. ${ }^{25} \mathrm{~A}$ concise exposition is presented below.

A suitable starting point for the analysis is the following (exact) multipolar Hamiltonian for the simple RET system comprising chromophores labeled $A$ and $B$ :

$$
H=H_{A}+H_{B}+H_{\text {int }}(A)+H_{\text {int }}(B)+H_{\text {rad }},
$$

where the first two terms are the unperturbed Hamiltonian operators for the chromophores, and the two $H_{\text {int }}$ operators represent interactions of the radiation field with $A$ and $B$. The final term, $H_{\text {rad }}$, is the radiation Hamiltonian which, because it, too, is an operator, is always part of the sum, even when no photons are present. It becomes immediately evident that no single term in Eq. (14.10) links $A$ with $B$; in other words, no static or longitudinal coupling occur. Hence, any form of coupling between the two chromophores has to be mediated by their individual interactions with the radiation field. This is an issue that it will be useful to revisit at the end of this section.

To continue, in the electric-dipole approximation, each $H_{\mathrm{int}}(\xi)$ is given by the usual dipole coupling formula

$$
H_{\mathrm{int}}(\xi)=-\sum_{\xi} \boldsymbol{\mu}(\xi) \cdot \mathbf{e}^{\perp}\left(\mathbf{R}_{\xi}\right) .
$$


In Eq. (14.11), the electric-dipole moment operator $\boldsymbol{\mu}(\xi)$ operates on matter states, and the transverse electric-field operator $\mathbf{e}^{\perp}\left(\mathbf{R}_{\xi}\right)$ on electromagnetic radiation states; $\mathbf{R}_{\xi}$ is the position vector of the chromophore labelled $\xi$. The electric field operator can be cast in the form of a mode summation, taken over optical wavevectors $\mathbf{p}$, and polarisations $\lambda$, which is usually written as follows: ${ }^{26}$

$$
\mathbf{e}^{\perp}\left(\mathbf{R}_{\zeta}\right)=\mathrm{i} \sum_{\mathbf{p}, \lambda}\left(\frac{\hbar c p}{2 \varepsilon_{0} V}\right)^{1 / 2}\left[\mathbf{e}^{(\lambda)}(\mathbf{p}) a^{(\lambda)}(\mathbf{p}) \mathbf{e}^{\mathrm{i}\left(\mathbf{p} \cdot \mathbf{R}_{\zeta}\right)}-\overline{\mathbf{e}}^{(\lambda)}(\mathbf{p}) a^{\dagger+(\lambda)}(\mathbf{p}) \mathbf{e}^{-\mathrm{i}\left(\mathbf{p} \cdot \mathbf{R}_{\zeta}\right)}\right] .
$$

In this mode expansion, $\mathbf{e}^{(\lambda)}(\mathbf{p})$ is the polarisation unit vector (an overbar denoting complex conjugate), $V$ is an arbitrary quantisation volume, and $a^{(\lambda)}(\mathbf{p})$ and $a^{\dagger(\lambda)}(\mathbf{p})$, respectively, are the photon annihilation and creation operators for the mode $(\mathbf{p}, \lambda)$. These ladder operators act on the radiation states through the relations

$$
\left.\begin{array}{l}
a^{(\lambda)}(\mathbf{p})|m(\mathbf{p}, \lambda)\rangle=\sqrt{m}|(m-1)(\mathbf{p}, \lambda)\rangle \\
a^{\dagger(\lambda)}(\mathbf{p})|m(\mathbf{p}, \lambda)\rangle=\sqrt{m+1}|(m+1)(\mathbf{p}, \lambda)\rangle
\end{array}\right\},
$$

and as such, engage $H_{\text {int }}$ in the fundamental processes of photon creation and annihilation.

The initial and final states for the RET process can be written as $\left|\varphi_{A^{*}} ; \varphi_{B} ; 0\right\rangle$ and $\left|\varphi_{A} ; \varphi_{B^{*}} ; 0\right\rangle$, respectively, each ket indicating the electronic states of the two chromophores and the number of photons present. For simplicity, vibrational levels are left out of the derivation, as they are readily incorporated at the end of the calculation. To effect the transition between the given system states, timedependent perturbation theory is applied with Eq. (14.11) as the perturbation operator. The overall process can be achieved only by applying this operator twice, necessarily signifying the creation and annihilation of a photon; the leading contribution to the quantum amplitude $M$ is therefore second order:

$$
M=\sum_{r} \frac{\left\langle f\left|H_{\mathrm{int}}\right| r\right\rangle\left\langle r\left|H_{\mathrm{int}}\right| i\right\rangle}{\left(E_{i}-E_{r}\right)}
$$

where $i, f$, and $r$ denote initial, final, and intermediate states of the system and $E$ signifies an energy. Since the photon will be unobservable and acts only in the capacity of effecting the coupling, it is termed a virtual photon, and its creation can take place at either $A$ or $B$. Two time orderings therefore arise: $(a)$ the virtual 
photon can be created at $A$ (effecting the decay of the donor-excited state) and subsequently annihilated at $B$ (effecting the acceptor excitation); $(b)$ the virtual photon may be created at $B$ (with the acceptor excitation) and annihilated at $A$ (with the donor decay). These two possibilities are illustrated by Feynman diagrams in Fig. 14.3, and by a state-sequence diagram in Fig. 14.4. The counterintuitive nature of case $(b)$ does not preclude its inclusion in the calculation; both here and in case (a), the mode expansion in the electric field operator Eq. (14.12) moreover requires summation over all optical frequencies. Physically, it can be understood that exact energy conservation is not imposed during the interval between creation and annihilation of the virtual photon, i.e., the ultrashort photon flight-time. This, a key feature of virtual photon behaviour, is entirely consistent with the time-energy Uncertainty Principle. When the whole system enters its final state, the balance of energy conservation is once again restored.

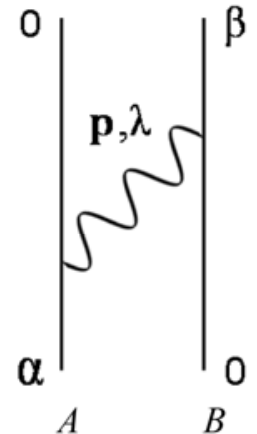

(a)

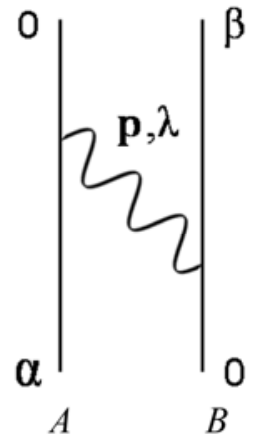

(b)

Figure 14.3 Feynman time-ordered diagrams corresponding to the two quantum amplitude contributions to RET, with time progressing upward. In (a) the virtual photon propagates from $A$ to $B$, and in (b) it moves from $B$ to $A$.

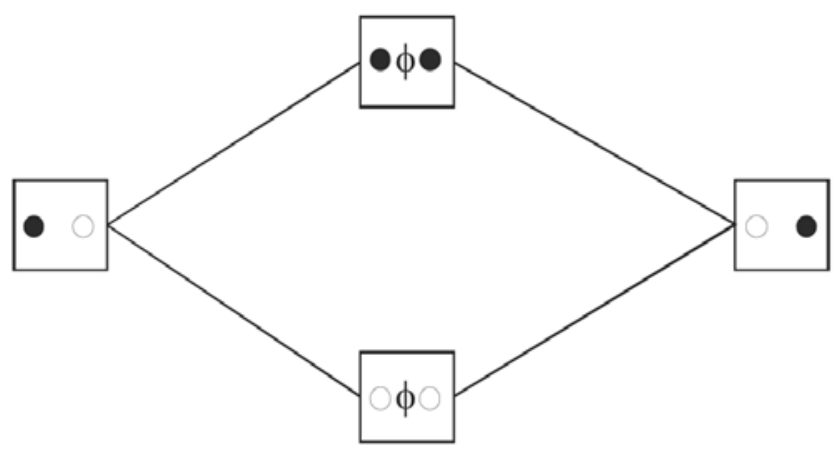

Figure 14.4 State-sequence diagram for RET, progressing from the initial system state of the left, through intermediate states, to the final state on the right. In each box, the two circles designate the states of $A$ and $B$, a filled circle denotes an electronic excited state, and an open circle the ground state; $\phi$ denotes the presence of a virtual photon. The lower pathway corresponds to the Feynman graph (a), and the upper pathway to (b) in Fig. 14. 3. 
Figures 14.3 and 14.4 readily facilitate determination of the two contributions to the quantum amplitude Eq. (14.14), which emerge as:

$$
\begin{aligned}
M_{i}=\sum_{\mathbf{p}, \lambda}[ & \frac{\left\langle\varphi_{A} ; \varphi_{B^{*}} ; 0\left|H_{\text {int }}\right| \varphi_{A} ; \varphi_{B} ; 1(\mathbf{p}, \lambda)\right\rangle\left\langle\varphi_{A} ; \varphi_{B} ; 1(\mathbf{p}, \lambda)\left|H_{\text {int }}\right| \varphi_{A^{*}} ; \varphi_{B} ; 0\right\rangle}{\hbar c k-\hbar c p} \\
& \left.+\frac{\left\langle\varphi_{A} ; \varphi_{B^{*}} ; 0\left|H_{\text {int }}\right| \varphi_{A^{*}} ; \varphi_{B^{*}} ; 1(\mathbf{p}, \lambda)\right\rangle\left\langle\varphi_{A^{*}} ; \varphi_{B^{*}} ; 1(\mathbf{p}, \lambda)\left|H_{\text {int }}\right| \varphi_{A^{*}} ; \varphi_{B} ; 0\right\rangle}{-\hbar c k-\hbar c p}\right],
\end{aligned}
$$

where $\hbar c p$ is the virtual photon energy and $k$ is defined through the equation for overall energy conservation:

$$
E_{A^{*}}-E_{A}=E_{B^{*}}-E_{B}=\hbar c k
$$

The wavevector and polarisation summations in Eq. (14.15) require substantial manipulation for evaluation by any of several standard techniques, detailed in the original papers and subsequent reviews. ${ }^{27}$ Implementing the necessary tensor calculus, the result emerges in a form concisely expressible as follows:

$$
M=\mu_{A i} V_{i j}(k, \mathbf{R}) \mu_{B j},
$$

using the convention of implied summation over repeated vector and tensor indices $i$ and $j$. In Eq. (14.17), the two transition dipole moments for the donor decay and acceptor excitation transitions are coupled by an E1-E1 coupling tensor defined by

$$
V_{i j}(k, \mathbf{R})=\frac{e^{i k R}}{4 \pi \varepsilon_{0} R^{3}}\left\{\left(\delta_{i j}-3 \hat{R}_{i} \hat{R}_{j}\right)-(\mathrm{i} k R)\left(\delta_{i j}-3 \hat{R}_{i} \hat{R}_{j}\right)-(k R)^{2}\left(\delta_{i j}-\hat{R}_{i} \hat{R}_{j}\right)\right\} \cdot(14
$$

It is of immediate interest to note that Eqs. (14.17) and (14.18) can be interpreted as the interaction of a transition dipole at $B$ with a retarded electric field $E$ produced by a transition dipole source at $A$, as given by $E_{j}=\mu_{A i} V_{i j}(k, \mathbf{R})$. This exactly correlates with the SI result delivered by classical electrodynamics: ${ }^{28}$

$$
\mathbf{E}=k^{2}(\hat{\mathbf{R}} \times \mu) \times \hat{\mathbf{R}} \frac{e^{i k R}}{4 \pi \varepsilon_{0} R}+[3 \hat{\mathbf{R}}(\hat{\mathbf{R}} \cdot \mu)-\mu]\left(\frac{1}{4 \pi \varepsilon_{0} R^{3}}-\frac{i k}{4 \pi \varepsilon_{0} R^{2}}\right) e^{i k R} .
$$

These are key results, the implications of which will now be examined in detail. 


\subsubsection{Near- and far-field behaviour}

Before considering the rate result that ensues from the above QED treatment, some features of physical significance can be identified from the quantum amplitude. First, it is important to note that for short-range distances where $k R$ «1 (signifying a length significantly smaller than the wavelength of the donor decay transition), Eq. (14.18) couples with the transition dipoles, as in Eq. (14.17), to deliver a result that equates exactly with the classical expression for the coupling, Eq. (14.19). The additional terms identified by QED, which come into play at larger distances, signify the intrinsic accommodation of retardationthese are features that reflect the finite time taken for $A$ and $B$ to interact, in accordance with relativistic principles of causality.

A second feature is manifest on effecting the tensor contraction $V_{i j} R_{i} R_{j}$ (again, with implied summation over $i$ and $j$ ), which identifies the longitudinal character of the coupling. It is evident that although the other terms in Eq. (14.18) deliver a finite result, the contribution from the last term is zero. Since the last term in Eq. (14.18) is in fact the long-range ( $k R » 1)$ asymptote, this result indicates that the far-field coupling is in fact fully transverse with respect to $R$, whereas near-field coupling is not (it has both transverse and longitudinal components). ${ }^{29}$ This behaviour is to be distinguished from the completely transverse character of the coupling field with respect to the virtual photon propagation vector, over all distances.

Recalling the sum over wavevectors in the electric field expansion Eq. (14.12), it can therefore be concluded that only virtual photons whose wavevector $\mathbf{p}$ is essentially parallel to the separation vector $\mathbf{R}$ remain significant for energy transfer as the donor-acceptor separation increases towards infinity. In contrast, the short-range or near-field behaviour is consistent with an involvement of virtual photons propagating in various directions. This can be understood as a manifestation of position-momentum quantum uncertainty, as illustrated in Fig. 14.5. In other words, as the distance $R$ increases, the virtual photon acquires an increasingly real character. ${ }^{29}$ To quote from a well-known

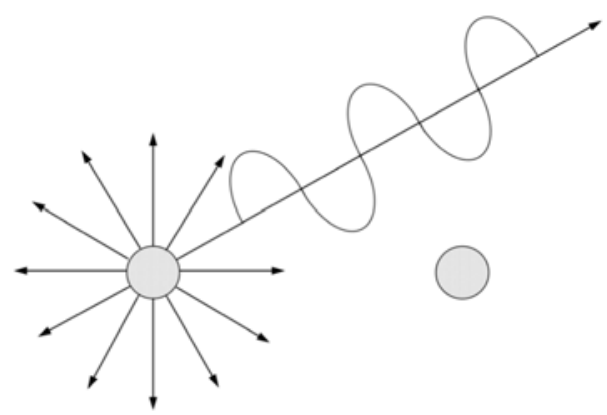

Figure $\mathbf{1 4 . 5}$ In the near field of an emitter-donor particle, virtual photons propagate in every direction, and quantum uncertainty allows their interaction with nearby absorber-acceptor particles; as the distance between emitter and absorber increases, the signal experienced by the latter is increasingly dominated by photons propagating directly towards it. 
textbook of elementary particle physics: 'In a sense every photon is virtual, being emitted and then sooner or later being absorbed. ${ }^{30}$ It is only the comparatively long lifetime, on a quantum timescale, of the photons we commonly observe that results in a disappearance of their virtual traits.

\subsubsection{Refractive and dissipative effects}

The above analysis, developed from Eq. (14.10), rigorously applies to a system of a single donor and acceptor with no other matter present. However, as most cases of interest concern the condensed phase, the electronic influence of surrounding or host material cannot in general be ignored, and we should include all atoms and molecules $(\xi)$ in a sum, writing:

$$
H=H_{A}+H_{B}+H_{\mathrm{int}}(A)+H_{\mathrm{int}}(B)+H_{\mathrm{rad}}+\sum_{\xi \neq A, B} H_{\xi}+\sum_{\xi \neq A, B} H_{\mathrm{int}}(\xi) \cdot(14
$$

The last three terms in Eq. (14.20) collectively represent an effective radiation field operator whose eigenstates signify modes in which the photons are 'dressed' by the electromagnetic influence of the host. Strictly speaking, these are polaritons, though the distinction is not important if one is dealing with frequencies at which the host is relatively transparent. By a lengthy development of theory, the effect of making this correction is that the coupling tensor Eq. (14.18) emerges in the following modified form, ${ }^{31}$ assuming Lorentz local field factors are assimilated into the expressions for the spectral functions $F_{A}(\omega)$ and $\sigma_{B}(\omega)$ :

$$
V_{i j}(k, \mathbf{R})=\frac{1}{n(\omega)^{2}} \frac{e^{i n(\omega) k R}}{4 \pi \varepsilon_{0} R^{3}}\left\{\begin{array}{l}
\left(\delta_{i j}-3 \hat{R}_{i} \hat{R}_{j}\right)-(\operatorname{in}(\omega) k R)\left(\delta_{i j}-3 \hat{R}_{i} \hat{R}_{j}\right) \\
-(n(\omega) k R)^{2}\left(\delta_{i j}-\hat{R}_{i} \hat{R}_{j}\right)
\end{array}\right\},(
$$

where $n(\omega)$ is the complex refractive index for electromagnetic radiation with an optical frequency $\omega=c k$.

\subsection{Features of the Pair Transfer Rate}

By use of the Fermi rule, ${ }^{32}$ the rate of energy transfer between a donor and acceptor pair has a quadratic dependence on the quantum amplitude. The quantum electrodynamical treatment of RET (discussed in the previous section) delivers a result that reveals important additional terms compared to the Förster Eq. (14.1), the extra terms arising from the influence of the bracketed terms, linear and quadratic in $n(\omega) k R$, in the quantum amplitude. ${ }^{21}$ The full result is conveniently represented as follows: ${ }^{33}$

$$
w=w_{\mathrm{F}}+w_{\mathrm{I}}+w_{\mathrm{rad}}
$$




$$
\left.\begin{array}{l}
w_{\mathrm{F}}=\frac{9 \kappa_{3}^{2} c^{4}}{8 \pi \tau_{A^{*}} R^{6}} \int F_{A}(\omega) \sigma_{B}(\omega) \frac{d \omega}{\omega^{4} n(\omega)^{4}} \\
w_{\mathrm{I}}=\frac{9 c^{2}}{8 \pi \tau_{A^{*}} R^{4}}\left(\kappa_{3}^{2}-2 \kappa_{1} \kappa_{3}\right) \int F_{A}(\omega) \sigma_{B}(\omega) \frac{d \omega}{\omega^{2} n(\omega)^{2}} \\
w_{\text {rad }}=\frac{9 \kappa_{1}^{2}}{8 \pi \tau_{A} R^{2}} \int F_{A}(\omega) \sigma_{B}(\omega) d \omega
\end{array}\right\} .
$$

In these expressions, the kappa orientational function of Eq. (14.2) is now generalised:

$$
\kappa_{j}=\left(\hat{\boldsymbol{\mu}}_{A} \cdot \hat{\boldsymbol{\mu}}_{B}\right)-j\left(\hat{\mathbf{R}} \cdot \hat{\boldsymbol{\mu}}_{A}\right)\left(\hat{\mathbf{R}} \cdot \hat{\boldsymbol{\mu}}_{B}\right)
$$

The first term of Eq. (14.22), $w_{\mathrm{F}}$, is the usual Förster rate, identical to Eq. (14.1) if the refractive index is taken as a constant. The second contribution, $w_{\mathrm{I}}$, is a correction that comes into play at distances beyond the near field, where the assumption $k R$ « 1 no longer holds. The third term, $w_{\text {rad, }}$ which dominates over both other contributions when $k R » 1$, equates exactly with the rate of acceptor excitation that results from the capture of a photon spontaneously emitted by the donor; i.e., a process of radiative transfer, its $R^{-2}$ dependence fittingly designating the familiar inverse square law. This radiative term manifests the long-range emergence of the coupling photon into a real character. Revisiting the underlying QED theory in order to disentangle the quantum pathways, recent work ${ }^{34}$ has also shown that this long-range behaviour is completely identifiable with the physically more intuitive sense of propagation for the virtual photon-case $(a)$ in Fig. 14.3, or the lower pathway in Fig. 14.4. In the same regime, the contribution from case $(b)$ 'backward' propagation drops off as $R^{-8}$. Nonetheless, it should be emphasised that $(a)$ and $(b)$ are equally important in the short range, where both run with $R^{-6}$.

The discovery that both Förster 'radiationless' and radiative coupling are components of a single mechanism that operates over all distances (beyond wavefunction overlap) has resulted in a paradigm shift in the understanding of RET, and is the reason that QED theory has been termed a unified theory. ${ }^{24}$ This theory is valid over a span ranging from the nanoscale up to indefinitely large distances; Förster energy transfer is the short-range asymptote and radiative transfer the long-range asymptote. Moreover, there is no competition between these processes, previously considered distinct, as they prove to be but aspects of a single coupling mechanism. However, the theory establishes more than this; it also addresses an intermediate range where neither the radiative nor the Förster mechanism is fully valid, because if $k R \sim 1$ to the nearest order of magnitude (suggesting distances in the hundreds of nanometers range), then all three terms in Eq. (14.23) contribute significantly. Although different kappa factors ( $\kappa_{3}$ and 
$\kappa_{1}$ ) characterise $w_{\mathrm{F}}$ and $w_{\text {rad }}$, both kappas are featured in the expression for $w_{\mathrm{I}}$. This is the main reason that the intermediate range behaviour has eluded experimental identification-because it is difficult to envisage any technical circumstances where its contribution could be directly isolated. However, an interesting interplay of distance and orientational factors does arise in connection with fluorescence polarisation measurements, as will be shown below.

\subsubsection{Distance dependence}

The three principal determinants of the transfer efficiency, as given by Eqs. (14.22)-(14.24), are the separation, mutual orientation, and spectral overlap of the donor-acceptor pair, the effect of each which will now be considered. First, for simplicity in beginning to consider the dependence on distance, let it be assumed that the donor and acceptor have isotropically averaged orientations and that the refractive index is taken as unity. It then follows that the distance dependence of the full rate expression Eq. (14.22) factorises out in the following form, ${ }^{24,35}$ which also identifies it with the tensor inner product of the QED coupling formula Eq. (14.18):

$$
A(k, R)=\frac{1}{8 \pi^{2} \varepsilon_{o}^{2} R^{6}}\left\{3+(k R)^{2}+(k R)^{4}\right\}=V_{i j}(k, \mathbf{R}) \bar{V}_{i j}(k, \mathbf{R})
$$

This excitation transfer function $A(k, R)$ is a scalar characterising the distance dependence of E1-E1 coupling in RET. The graph shown in Fig. 14.6 exhibits a log-log plot of the function over the range of $1 \mathrm{~nm}$ to $1 \mu \mathrm{m}$, for a value of $k=9 \times$ $10^{6} \mathrm{~m}^{-1}$ (corresponding to a wavelength at the red end of the visible region). This figure gives a readily comprehensible representation of the Förster behaviour and 'radiative' transfer as short- and long-range asymptotes, respectively, as shown by the change in gradient between the short- and long-range regions.

Most applications of RET relate to the Förster regime, i.e., systems in which energy-transfer steps occur between chromophores separated by less than the Förster distance, and therefore almost certainly within the short-range regime, $k R$ «1. Systems in which the mean transfer distance falls in the long-range regime, $k R » 1$, necessarily require the optically relevant species to be present in low concentrations; moreover, any diffusion processes that could produce transient short-range donor-acceptor juxtapositions should have a timescale significantly longer than the donor decay time, or else diffusion-limited Förster transfer would result. The radiative transport that ensues in the latter case (in a variety of systems, such as dilute dye solutions) leads into a distinct branch of the theory in which multiple scattering must also be considered; a detailed account is given by Berberan-Santos et al. ${ }^{36}$ The possibility of experimentally verifying the general form of distance dependence given by Eq. (14.25), and, in particular, identifying the intermediate $R^{-4}$ term, remains a currently unachieved but tantalising prospect. 


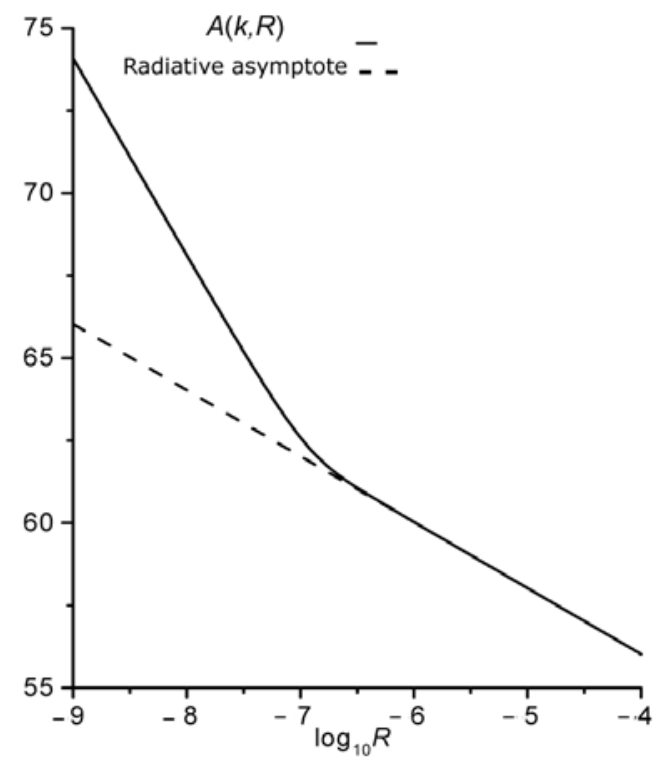

Figure 14.6 Log-log plot of the RET excitation transfer function $A(k, R)$, as defined by Eq. (14.25), against the donor-acceptor distance $R$ (in nanometers) for $k=9 \times 10^{6} \mathrm{~m}^{-1}$.

\subsubsection{Orientation of the transition dipoles}

The $\kappa$ factors in Eq. (14.23), which depend on the mutual orientations of the donor and acceptor (see Fig. 14.7), represent another important facet of the energy transfer. ${ }^{37-39}$ Certain orientations reduce the rate of transfer to zero; for others, they effect an 'enhancement' of the energy transfer to its maximum possible rate. It is worth noting (since it is a fact that is not infrequently misreported), that energy transfer may be permissible even when the relevant donor and acceptor transition moments are at right angles to each other, if the second term in Eq. (14.24) is nonzero. In fact, the only case in which the transfer is necessarily forbidden, given the condition $\mu_{A} \perp \mu_{B}$, is when one of these transition dipoles is also orthogonal to $\mathbf{R}$. In this situation, all three terms of Eq. (14.23) vanish.

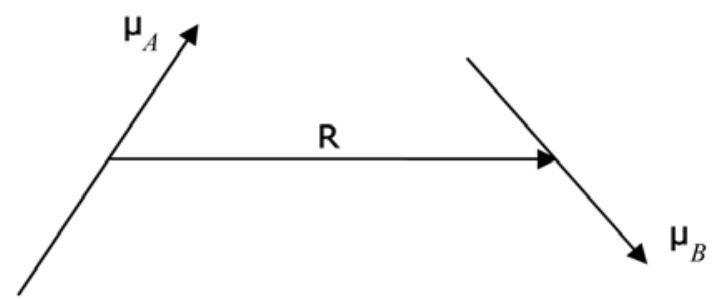

Figure 14.7 Typical noncoplanar orientations of the donor and acceptor transition dipoles, relative to the displacement vector. 
Other factors can complicate the orientation dependence of RET. One is the fact that in any system that is to an extent fluid or disordered, the relative orientation of all donor-acceptor pairs may not be identical. It has already been noted that in the isotropic case (having completely uncorrelated orientations), the $\kappa_{3}^{2}$ factor in $w_{\mathrm{F}}$ averages to $2 / 3$; however, when a degree of orientational correlation is present, other results are possible (in the overall range of $0-4$ ), the exact value characterising the detailed form of the angular distribution. Secondly, it can happen that either the donor or the acceptor transition moment is not unambiguously identifiable with a particular direction within the corresponding chromophore frame of reference. Specifically, the electronic transition may then relate to a degenerate state, as can occur with square planar complexes, for example. Alternatively, very rapid but orientationally confined motions might occur. The considerable complication that each of these effects brings into the trigonometric analysis of RET has been extensively researched and reported by van der Meer. ${ }^{2}$

It can at first sight be surprising to discover that the relative orientation of transition dipoles is manifest in readily discernible polarisation effects, even in media where the donor and acceptor orientations are uncorrelated. For example, when a single molecule in solution absorbs and then fluoresces, the angle $\alpha$ between the absorption and emission transition moments can be deduced from a determination of the fluorescence anisotropy, defined as ${ }^{12,13}$ :

$$
r=\frac{I_{\|}-I_{\perp}}{I_{\|}+2 I_{\perp}},
$$

where $I_{\|}$and $I_{\perp}$ respectively designate the intensities of fluorescence parallel and perpendicular to the polarisation of the excitation beam. It is relatively straightforward to show that

$$
r_{0}=\frac{1}{5}\left(3 \cos ^{2} \alpha-1\right)
$$

the subscript on the left indicating that no transfers of energy are involved. The value $r_{0}=0.4$ ensues when the absorption and emission moments are parallel. In a two-chromophore donor-acceptor system, if the pair were to be held in a fixed mutual orientation but could freely tumble as a pair, the result [Eq. (14.27)] would still be applicable, provided that within each chromophore there were parallel excitation and decay transition moments; the calculated value of $\alpha$ would then signify the angle between those differently oriented directions in the donor and acceptor.

If, however, the donor and acceptor are free to rotate independently, then the act of energy transfer significantly reduces the extent of the fluorescence anisotropy. It has long been known that one transfer step reduces the anisotropy 
to a value of $\frac{1}{25} r_{0}$, at least for conventional Förster transfer. However, the complete result, which first emerged from a detailed analysis based on the retarded coupling tensor Eq. (14.21), is as follows: ${ }^{40}$

$$
r_{1}=\frac{r_{0}}{25}\left(\frac{7 \overline{k^{4} R^{4}}+\overline{k^{2} R^{2}}+3}{\overline{k^{4} R^{4}}+\overline{k^{2} R^{2}}+3}\right)
$$

in which the overbars signify distributional averages over the $k$ and $R$ values for the transfer. A graph of the function $r_{1} / r_{0}$ is shown in Fig. 14.8. Clearly in the long-range asymptote corresponding to radiative transfer between the donor and acceptor, the anisotropy $r_{1}$ takes the value $\frac{7}{25} r_{0}$. The result is appealing because it manifests so dramatically the onset of retardation effects.

\subsubsection{Spectral overlap}

Finally, each component in the overall RET rate Eq. (14.23) involves an integral over a frequency-weighted product of the donor emission and acceptor absorption profiles. As such, it is not only the peak frequencies or wavelengths for emission and absorption that dictate the energy transfer conditions; so do the spectral shapes and bandwidths. Such features assume particular significance in connection with the multistep processes that take place in energy-harvesting materials. This subject will be further developed in that connection, in Section 14.5.1.

Most theoretical work on the spectral overlap addressing the Förster regime, ignores the dispersion of the refractive index that is featured in $w_{\mathrm{F}}$. General analytical expressions for the frequency-weighted overlap have been determined for several cases of spectral line-shape. Principal among these is the Gaussian case, which fits reasonably well the line profile of many molecular absorption and emission processes in the condensed phase, where the high densities of vibrational levels in the ground and excited electronic states produce a

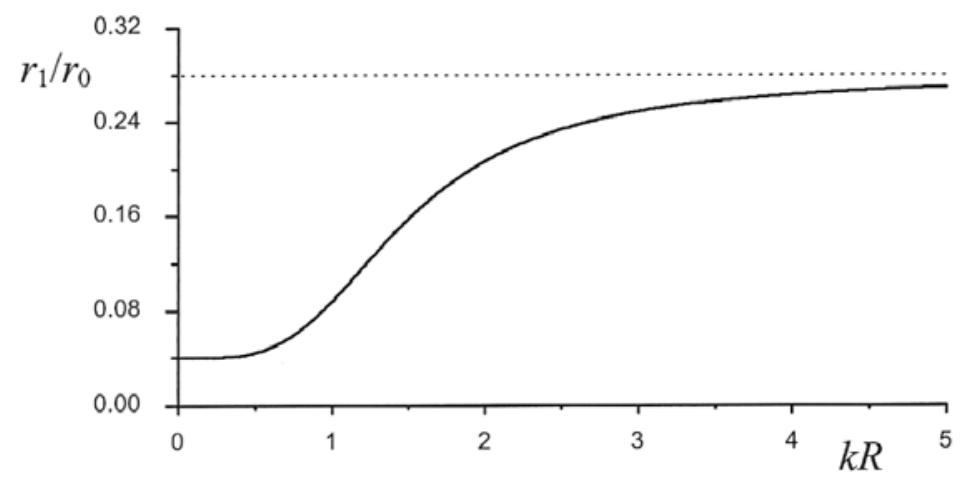

Figure 14.8 Rise in fluorescence anisotropy as a function of the distance between orientationally uncorrelated donor and acceptor particles. 
broadening that is similar in effect to a stochastic perturbation. For spectra that depart markedly from a completely symmetric Gaussian shape, a better fit is often afforded by a log-normal distribution. Typically, systems with the latter form of line-shape exhibit a sharper rise to the maximum on the high-frequency side of the absorption spectrum, and on the low-frequency side of the emission spectrum, together retaining the familiar ideal of a 'reflection' relationship between absorption and emission spectra.

\subsection{Energy Transfer in Heterogeneous Solids}

A wide variety of materials can be engineered to exhibit photoactive properties based on RET. In this section, three types of systems are chosen and discussed to illustrate this range: doped solids, quantum dots, and multichromophore complexes. Not surprisingly, quite different attributes and applications are associated with each system type.

\subsubsection{Doped solids}

In optical materials dilutely doped with transition metals, RET represents a diffusive mechanism that moderates other processes such as stimulated emission (as another example, it influences laser efficiency). RET also plays an important role in the photophysics of rare-earth (lanthanide)-doped crystals, glasses, and fibers. These optically dilute materials display spectral features in the visible and near-visible regions that owe their origin to $f$-shell electronic transitions in the dopant. $^{41}$ The narrow linewidth of the energy levels, together with the coincidences of spacing between levels in different lanthanides, afford excellent opportunities for the design of materials that invoke not only conventional RET, but also higher-order effects. ${ }^{42}$ A variety of proposals by Dexter ${ }^{43}$ and Bloembergen $^{44}$ in the 1950 s built on the premise of deploying RET to relay excitation between lanthanide ions. Following the development of lasers, the predicted higher-order effects were quickly brought to experimental fruition. Principal among the latter processes are stepwise or cooperative up-conversion, sensitisation, and down-conversion. ${ }^{45,46}$

The term up-conversion signifies processes in which low-frequency radiation is converted to a higher frequency, the output itself often being used as a basis for laser emission. In common with parametric processes involving the direct conversion of electromagnetic radiation in optically nonlinear crystals, the RETbased mechanism furnishes an output in which each photon is created at the expense of two input photons. In contrast to parametric processes, however, the input photons are not required to arrive simultaneously. The consequence is that the effect is achievable at lower intensities. The simplest scheme is one in which transfers of energy from two initially excited but spatially isolated donors, $A^{*}$ and $B^{*}$, promote an acceptor $C$ to a state with approximately twice the energy of the first excitation. One possibility is a stepwise process in which the twin donors sequentially deliver excitation energy to the acceptor, as shown in Fig. 14.9. In 
some of the literature, this is referred to as APTE (Addition de Photons par Transferts d'Energie). ${ }^{47}$ The mechanism can operate even when there is no suitable energy level to accommodate the initial transfer step. To satisfy the principle of energy conservation, the mechanism requires concurrent operation of the RET processes accommodated by the involvement of a virtual level in the acceptor; this latter process is often known as cooperative up-conversion. Chua and Tanner have shown how the theory can be extended to distance regimes where long-range radiative transfer is dominant. ${ }^{48}$

The QED theory of APTE up-conversion invokes fourth-order timedependent perturbation theory, as befits the need to create and annihilate two virtual photons to effect the necessary couplings. Calculations lead to a quantum amplitude comprising three types of terms, each itself comprising 24 different contributions based on the allowed pathways through a state-sequence diagram, ${ }^{49}$ as illustrated in Fig. 14.10. Of the three quantum amplitude components, one is designated cooperative to signify that each donor loses its energy directly to the acceptor; the other two are termed accretive, signifying that one donor passes its energy to the other, from which the sum energy is conveyed to the acceptor. Two cases arise due to the choice of the alternative roles played by each donor. The results are as follows: ${ }^{50-52}$

$$
\left.\begin{array}{l}
M_{i}^{\mathrm{coop}}=\mu_{A i} V_{i j}(k, \mathbf{R}) \alpha_{C j k}(-\omega,-\omega) V_{k l}\left(k, \mathbf{R}^{\prime}\right) \mu_{B l} \\
M_{i}^{\mathrm{acc} 1}=\mu_{A i} V_{i j}\left(k, \mathbf{R}^{\prime \prime}\right) \alpha_{B j k}(2 \omega,-\omega) V_{k l}\left(2 k, \mathbf{R}^{\prime}\right) \mu_{C l} \\
M^{\mathrm{acc} 2}=\mu_{B i} V_{i j}\left(k, \mathbf{R}^{\prime \prime}\right) \alpha_{A j k}(2 \omega,-\omega) V_{k l}(2 k, \mathbf{R}) \mu_{C l}
\end{array}\right\},
$$

where $\omega=c k$, once again, $k$ is as defined by Eq. (14.16), and each $\mu$ is a transition moment; $\alpha(-\omega,-\omega)$ is a two-photon absorption tensor, $\alpha(2 \omega,-\omega)$ is formally an electronic anti-Stokes Raman tensor, and the three mutual displacement vectors are defined by $\mathbf{R}=\mathbf{R}_{C}-\mathbf{R}_{A}, \mathbf{R}^{\prime}=\mathbf{R}_{C}-\mathbf{R}_{B}$, and $\mathbf{R}^{\prime \prime}=\mathbf{R}_{B}-\mathbf{R}_{A}$.

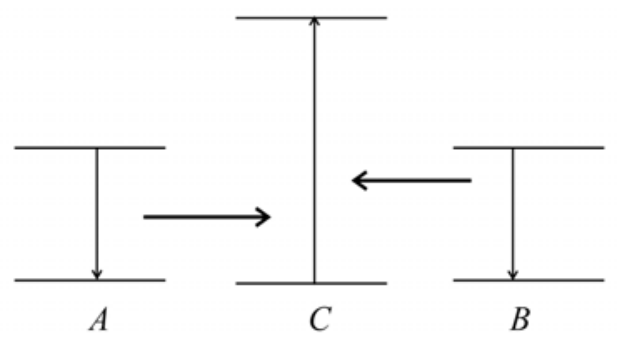

Figure 14.9 Diagram representing cooperative up-conversion. 
For simplicity (conforming to the usual experimental case) it is assumed that $A$ and $B$ are chemically identical. From Eq. (14.29), the quadratic dependence of the up-conversion rate produces a result with three 'diagonal' and six 'offdiagonal' terms, as well as a complicated dependence on the dopant concentrations $C$ of the donor and acceptor ions. For example, the third term of Eq. (14.29) contributes a diagonal rate term that in the short range runs as $R^{\prime-6} R^{\prime \prime}$, producing a sum expressible as $\eta C_{A} C_{B}^{2} C_{C}$ (with, for example, $\eta=$ 64.39 for a regular lattice of cubic symmetry). ${ }^{52}$

Similar principles operate in sensitisation processes. ${ }^{53}$ Here, the transfer of excitation from a donor ion $A^{*}$ to an acceptor $C$ engages a bridging species $B$, without which the transfer is ineffective. Once again, the term 'up-conversion' is common for such observations, but 'sensitisation' distinguishes it from the pooling processes described above. The other principal case of interest, degenerate down-conversion or quantum cutting, is a phenomenon in which excitation is simultaneously conveyed to two acceptors $A$ and $B$, from a single excited donor $C^{*}$, each transfer carrying approximately half of the energy of the donor excitation. ${ }^{54}$ Thus, for example, an initial excitation of VUV radiation can lead to the emission of visible light. Systems exhibiting such effects represent the fulfillment of very early suggestions by Dexter of systems that can exhibit quantum yields greater than unity. ${ }^{43}$

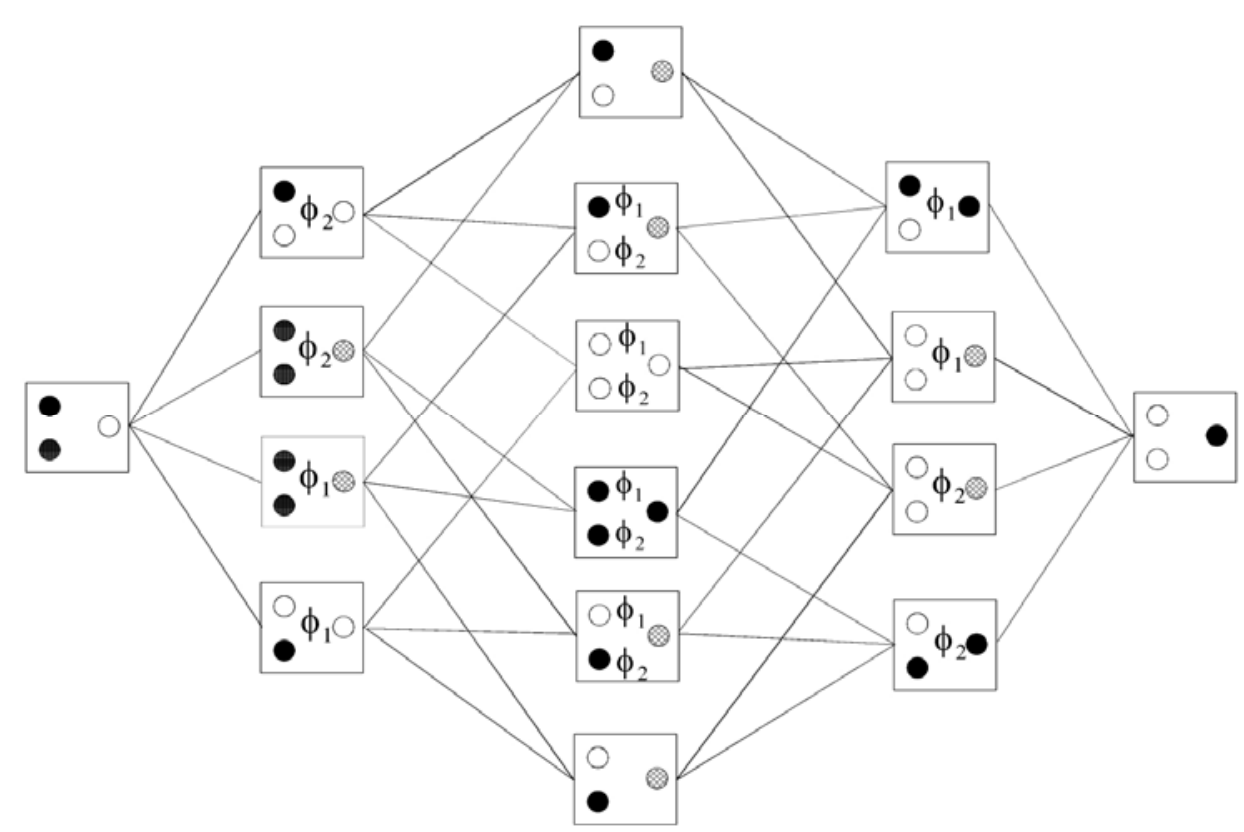

Figure 14.10 State-sequence diagram for cooperative up-conversion, accommodating 24 quantum amplitude contributions. In each box, the two circles on the left denote donors and that on the right, an acceptor. Grey circles designate virtual states; black and white circles denote excited and ground states. 


\subsubsection{Quantum dots}

There is growing interest in a variety of schemes that are based on the transfer of excitation between adjacent quantum dots, ${ }^{55-64}$ and are now being envisaged for performing quantum computation. Such proposals generally aim to exploit the discrete, size-tunable, and intense character of quantum dot exciton transitions, as well as the fact that these processes can be switched very rapidly using optical excitation. Moreover, it proves possible to code additional information by the use of circularly polarised excitation, since this enables specific exciton spin states to be populated. ${ }^{65}$ This important question thus arises: Can the spin state of an exciton be transmitted or flipped through RET between suitably organised quantum dots? The answer is provided by a QED analysis ${ }^{66}$ that separately identifies those contributions to the RET quantum amplitude as corresponding to left- and rightcircular polarisations of the coupling virtual photons. The resulting plots in Fig. 14.11 show the effect of rotating one quantum dot relative to another (assuming the electronic coupling to be real). When the transition moments are parallel, the exciton orientation is transmitted unchanged from one quantum dot to another; when they are antiparallel, the geometry causes the exciton spin to flip. Energy migration down a column of quantum dots oriented in a common direction therefore proceeds with a full retention of spin orientation.

One advantage of the QED treatment of the problem is that not only the short-range but also the long-range behaviour is identified, even though the latter is of less interest from an application viewpoint. In the long-range asymptote, it is clear that angular momentum must be conserved about the propagation direction of the photon, which coincides with the mutual displacement vector of adjacent quantum dots and hence, the local columnar morphology. Thus, it transpires that the same feature operates in the technically important near-zone region, even though the coupling cannot in this case be ascribed to real photon propagation. Energy migration down a column of quantum dots with a common orientation preserves spin information, absolutely; the observation of spin flipping between alternately inverted quantum dots is another manifestation of the same principle.
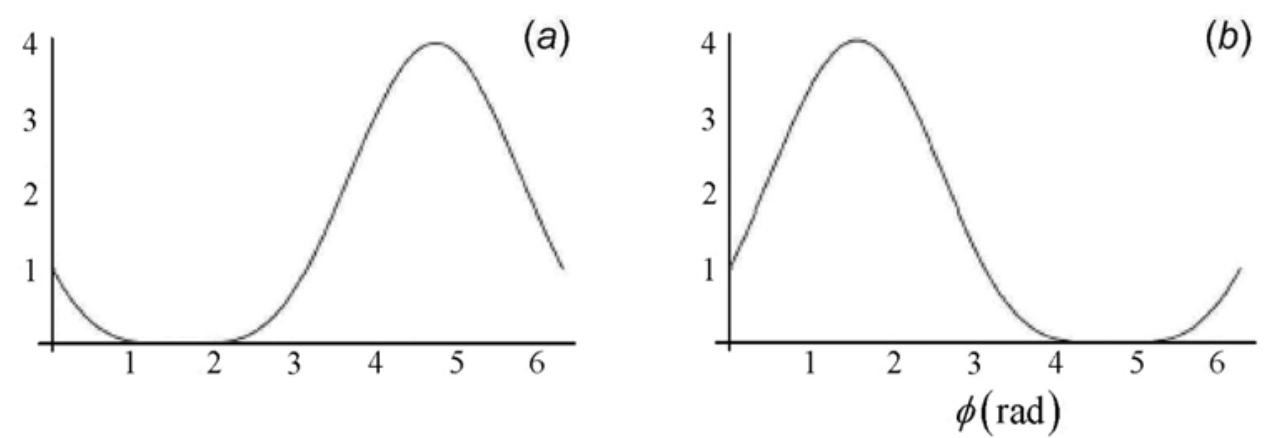

Figure 14.11 Quantum dot energy transfer. Variation of (a) spin antiparallel and (b) spin parallel transfer functions as a function of relative orientations. 


\subsubsection{Multichromophore complexes}

RET is an extremely significant process in the operation of photosynthetic and biomimetic light-harvesting materials. ${ }^{67-72}$ The photosynthetic systems of purple bacteria, in particular, have been extensively studied and characterised, and the emulation of their high efficiency is a key goal in the development of new polymer-based materials.

In order to most effectively utilise the sunlight that falls on them, photosynthetic organisms have a system of antenna complexes surrounding the reaction centres where photosynthesis takes place. ${ }^{73,74}$ The complexes absorb sunlight and the acquired energy migrates towards the reaction centre by a series of short-range, radiationless energy-transfer steps (see Fig. 14.12). In the overall migration of energy from the site of its initial deposition to the site of its chemical action, a spectroscopic gradient (see Section 14.5.1) is one of the key directional principles obviating random diffusion. Energy is quickly and efficiently directed towards a reaction centre. Not only does this allow an organism to harvest light incident on a large surface area, but by pooling energy from a large number of antenna chromophores, energy of a higher equivalent frequency can be produced. This is essential, since the majority of the incident light from the sun has too low a frequency for its individual photons to drive photosynthesis.

It is not only the spectroscopic properties of the chromophores that determine the character and direction of energy flow; the chromophore positioning and orientation are also important. Two-dimensional optical spectroscopy can unveil the intricate interplay between spectral and spatial overlap features in lightharvesting complexes, as beautifully exhibited in a recent study on

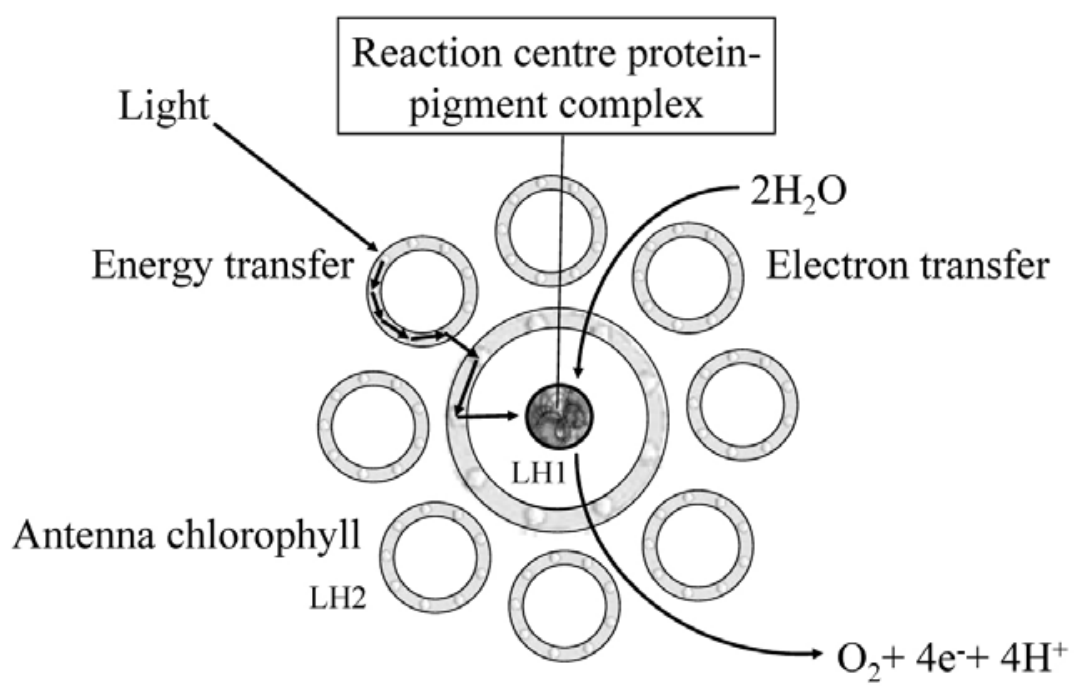

Figure 14.12 Energy flow in a bacterial photosystem for the oxidation of water. The outer rings of light-harvesting complex $\mathrm{LH} 2$ surround one inner ring of $\mathrm{LH} 1$ complex, near the middle of which is the reaction centre. 
bacteriochlorophyll. ${ }^{75}$ Interrogating the system with a sequence of ultrashort laser pulses, the optical response of the sample reveals linear absorption processes and couplings between chromophores, as well as dynamical aspects of the energy transfer. The results show that excitation relocation does not proceed simply by stepwise transfer from one energy state to another of nearest energy; it depends on strong coupling between chromophores, determined by the extent of their spatial overlap. Thus, excitation relocation may involve fewer intermediary chromophores than might otherwise be expected.

The efficiency of photosynthetic units has encouraged the design of a variety of synthetic light-harvesting systems. ${ }^{76-79}$ The materials that have received the most attention are dendrimers, ${ }^{80,81}$ macromolecules consisting of molecular units repeatedly branching out from a central core. Designed to act as an excitation trap, dendrimers are exemplified by the structure shown in Fig. 14.13. The outward branching leads to successive generations of structures, each with an increased number of peripheral antenna chromophores. In ideal cases, the requisite spectroscopic gradient is established through chemically similar chromophores in generationally different locations. ${ }^{82,83}$ The most recent work on dendrimers has utilised branching motifs of threefold and fourfold local symmetry, based on tri-substituted benzene ${ }^{84}$ and porphyrin rings, ${ }^{85}$ respectively.

\subsection{Directed Energy Transfer}

There are a number of different ways in which a vectorial character can be produced or enhanced in RET. ${ }^{86}$ In the operation of multichromophore complexes in particular, it is highly important to expedite the delivery of energy (acquired through photoabsorption) to an appropriate location, rather than allowing it to be subject to a long-path random walk with the attendant likelihood of dissipation as heat. Although the mechanisms for controlling energy flow are only just beginning to receive the full attention they deserve, a number of important principles have already been identified.

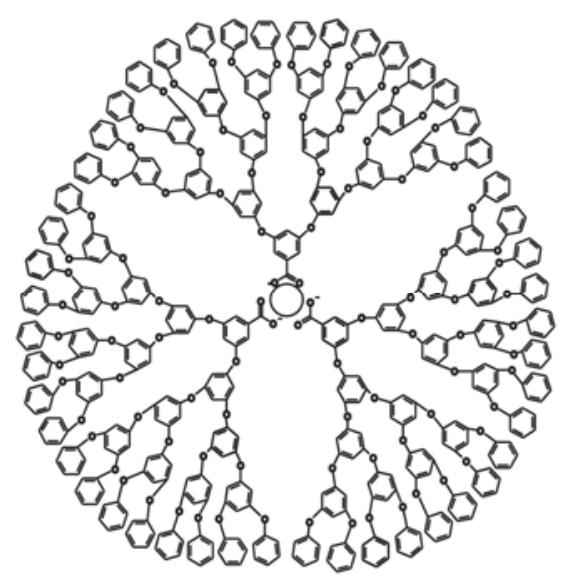

Figure 14.13 Fifth-generation polyphenylether dendrimer. 


\subsubsection{Spectroscopic gradient}

In the context of energy-harvesting materials, it has been shown that any pair of chromophores that plays the role of donor and acceptor will often do so as part of a sequence in which its donor unit acquires energy, and the acceptor passes that energy further onward, through preceding and subsequent RET couplings. Commonly, the energy passes onward through a sequence of chromophores exhibiting progressively longer wavelengths of absorption, because they differ either in their molecular structure, or in their local electronic environment (the latter being a particularly prominent feature of photobiological systems). This progression through increasingly red-shifted chromophores, known as the spectroscopic gradient or 'energy funnel,' is the reason for the directed character of energy capture by the system.

In general, the excitation of each successive donor populates a vibrationally excited level within its electronic excited state, from which a degree of vibrational relaxation immediately occurs. The result is a slight degradation of the energy with each transfer event. Small enough not to be of major concern in energy efficiency terms, this feature of multistep energy migration is indeed the factor that largely determines its directed character. This is because, at each step, 'forward' transfer is favored over 'backward' transfer. The single parameter that effectively determines the extent of this favor is the ratio of spectral overlaps for forward and backward transfer, a parameter that exerts a significant influence on the overall trapping efficiency of any energy-harvesting complex. For example, in the case of dendrimers, the relative propensities for forward and backward transfer between different generation shells is a key factor in determining the directedness of energy flow towards the acceptor core.

The overall spectroscopic gradient experienced on the passage of excitation through a multichromophore system crucially depends on the relative propensities for the forward and backward transfer of energy at each individual step, as determined by the optical and photophysical properties of the relevant units acting as donor and acceptor. ${ }^{87}$ To quantify such relative propensities, it is therefore convenient to introduce a dimensionless efficiency parameter $\varepsilon$.

$$
\varepsilon=\frac{\tau_{B^{*}}}{\tau_{A^{*}}} \times \frac{\int F_{A}(\omega) \sigma_{B}(\omega) \omega^{-4} d \omega}{\int F_{B}(\omega) \sigma_{A}(\omega) \omega^{-4} d \omega} .
$$

The integrals in the numerator and denominator of Eq. (14.30) are respectively defined as the 'forward' and 'backward' spectral overlaps. Their role in determining the directionality of energy transfer at the pair-chromophore level is illustrated by Fig. 14.14. The peak frequencies of $\sigma_{B}, F_{A}, F_{B}$, and $\sigma_{A}$ are $\omega_{1}, \omega_{2}$, $\omega_{3}$, and $\omega_{4}$, respectively, and each fluorescence peak is duly red shifted with respect to its absorption counterpart. In the forward transfer process, the excitation of $A$ is followed by intramolecular vibrational redistribution (IVR) that dissipates part of the acquired energy. Following energy transfer, the same feature is observed in $B$. On comparing this process with the inverse transfer, $B$ to 
$A$, it is clear that the forward case is favored because $\omega_{4}-\omega_{3}$ exceeds $\omega_{2}-\omega_{1}$ ( $\delta_{2}>\delta_{1}$ in Fig. 14.14); in general, it follows that the larger the value of $\delta_{2}$, and the smaller the value of $\delta_{1}$, the higher the relative efficiency. Two parameters expediently quantify the extent of the spectroscopic gradient: the absorption shift (the frequency displacement of the acceptor absorption maximum relative to that of the donor band), $\omega_{\mathrm{G}}=\omega_{4}-\omega_{1}$ and the corresponding fluorescence shift, $\omega_{\mathrm{G}}^{\prime}=\omega_{2}-\omega_{3}$.

The general expression in Eq.(14.30) has been analysed in detail for a variety of complex spectral line-shapes, and the results are reported elsewhere. ${ }^{87}$ Even the simplest case has interpretive value: when the relevant spectral functions are considered to be Gaussians of similar width (FWHM $\Delta \omega$ ) and height, the result for $\varepsilon$ is expressible as follows;

$$
\varepsilon \cong e^{2 k\left(\delta_{2}^{2}-\delta_{1}^{2}\right)} \cong e^{2 k \omega_{\mathrm{G}} \omega_{\mathrm{S}}},
$$

where $k=4 \ln 2(\Delta \omega)^{-1}$ and in the expression on the right, written in terms of the Stokes shift, $\omega_{\mathrm{s}}=\omega_{4}-\omega_{2}=\omega_{1}-\omega_{3}=\delta_{2}-\delta_{1}$, the approximation is based on making $\omega_{\mathrm{G}}$ and $\omega_{\mathrm{G}}^{\prime}$ equal. Notably, the ensuing result shows precisely the same functional dependence on the spectroscopic gradient and the state shift; the conclusion is that both properties are equally important in determining the directedness of the energy transfer.

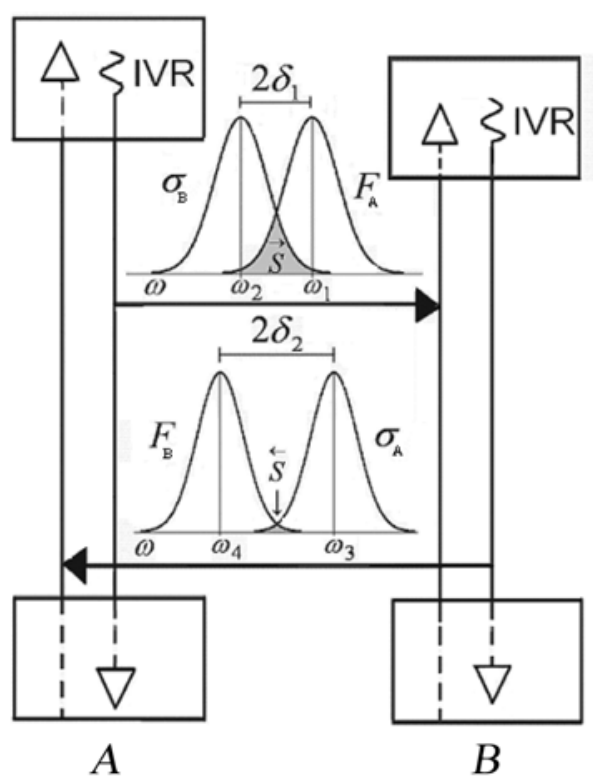

Figure 14.14 Energetics and spectral overlap features for forward and backward energy transfer between donor $A$ and acceptor $B$. 


\subsubsection{Influence of a static electric field}

The process of energy transfer can be significantly modified by interaction with a static electric field. ${ }^{88}$ At a simple level, the influence can be understood as a consequence of effecting shifts in the electron distributions of the interacting chromophores, producing modified transition moments. In more detail, new contributions to the RET quantum amplitude [Eq. (14.15)] arise, featuring additional interactions with the static field. The most significant corrections entail linear coupling of the field with $A$ or $B$, while higher-order correction involves field coupling with both chromophores. Significantly, when the static field engages with either the donor or the acceptor transition, different selection rules are invoked: those formally associated with a two-quantum transition. For example, if both the donor and acceptor transitions are electric-dipole-forbidden, only the higher-order interaction can mediate the energy transfer (except, conceivably, by involving a much weaker, higher-order multipole). The physical significance is that, in such a system, energy may not transfer without the presence of the static field; in a suitably designed system, the field-induced mechanism therefore allows electrically switchable control over the delivery of energy to the acceptor. ${ }^{86}$

\subsubsection{Optically controlled energy transfer}

The current pace of development in nanofabrication techniques has promoted an increasing interest in the specific effects of donor and acceptor placement in nanoscale geometries and periodic structures. However, the possibility of influencing the operation of RET by an optical field, through the input of an offresonant auxiliary beam of laser radiation, has only very recently begun to receive consideration.

Attention was first focused on amplification effects that might be observed in systems where energy transfer can occur without an auxiliary beam. It was shown that, at the levels of intensity currently available from mode-locked solid state lasers, significant enhancements of the transfer rate could be expected. ${ }^{89}$ Although the initial work anticipated effects that could be manifest in any donor-acceptor system, interest has subsequently refocused on structures tailored to exploit the laser-assisted phenomenon. Specifically, consideration has turned to systems in which each donor-acceptor pair has optical properties that satisfy the spectral overlap condition, but for which RET is designedly precluded by a customised geometric configuration. ${ }^{90}$ For example, as was observed in Section 14.3.2, both short- and long-range RET is forbidden when the donor and acceptor undergo electric dipole transitions whose transition moments are perpendicular both to each other, and to the donor-acceptor displacement vector. Through an optically nonlinear mechanism of optically controlled resonance energy transfer (OCRET), it transpires that the throughput of nonresonant laser pulses can facilitate energy transfer under such conditions where it would otherwise be rigorously forbidden; the system thus functions as an optical transistor, with excitation throughput switched on by the auxiliary beam. The laser systems most capable of delivering 
the necessary levels of irradiance prove to be precisely those that will also offer directly controllable ultrafast speeds of switching.

The mechanism for OCRET invokes fourth-order time-dependent perturbation theory. Each interaction is linear in the electric dipole interaction operator and thus, involves the absorption or emission of a photon. Specifically, in addition to the two virtual photon events (creation and annihilation) of normal RET, this process involves the absorption and the stimulated re-emission of a photon from the throughput off-resonant laser light. In common with the virtual photon interactions, each of the real photon events may occur at either the donor or the acceptor. In general, all four of the resulting possible combinations contribute to the overall quantum amplitude; moreover, each has 24 different time orderings associated with it. Figure 14.15 illustrates two of the 96 Feynman diagrams that arise. Again, the state-sequence method ${ }^{49,91}$ represents a considerably more tractable basis for the QED calculations that are necessary to deliver an equation for the energy transfer rate.

Recently, detailed calculations have been performed on a prototype implementation of OCRET in planar nano-arrays (Fig. 14.16). The results, illustrated in Fig. 14.17, give encouragement that the mechanism affords a realistic basis for fabricating a configuration of optical switches with parallel processing capability, operating without significant cross-talk. The analysis also supports a view that a nano-array OCRET system may, in the longer term, come to represent a new and significant channel of progress towards reliable systems for use in optical computing and communications routing. ${ }^{92}$

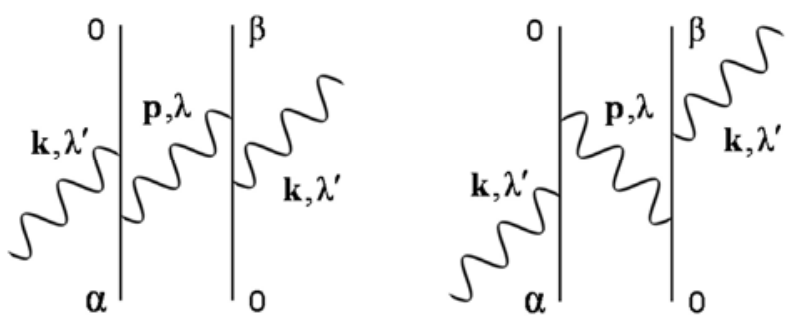

Figure 14.15 Two of the 96 Feynman time-ordered diagrams for OCRET.

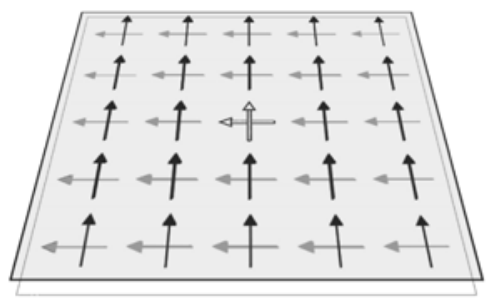

(a)
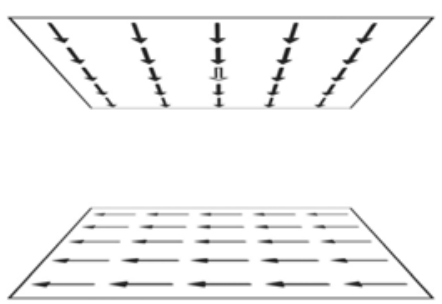

(b)

Figure 14.16 Two views of the nano-array structure: (a) from above and (b) from between the layers with their separation exaggerated for clarity. Black arrows represent donor transition dipoles of the upper array and grey the acceptor transition dipoles of the lower array; open arrows represent the excited donor and its corresponding ground-state acceptor. 


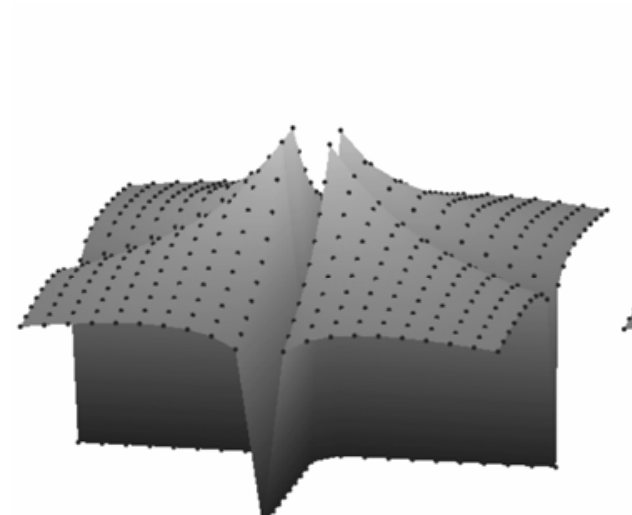

(a)

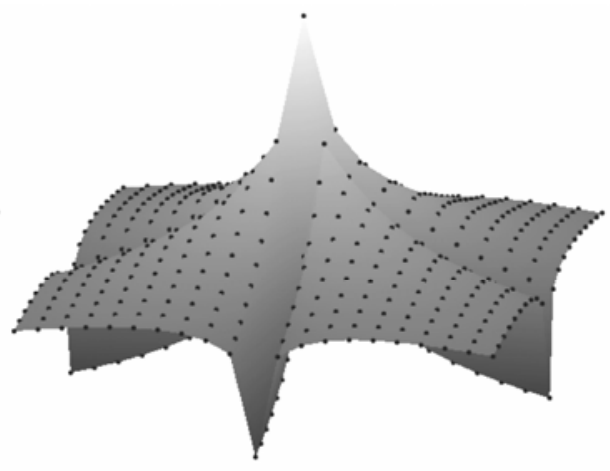

(b)

Figure 14.17 Contour graphs depicting, on a logarithmic scale, the probability of energy transfer to the array of acceptors (a) in the absence and (b) in the presence of laser light. The efficiency of energy transfer is represented by the vertical scale and the dots indicate the spatial locations of each acceptor.

\subsection{Developing Applications}

The mechanism of RET is involved in an ever-broadening range of applications, only some of which have been covered in this chapter. As mentioned earlier, the practice of measuring the fluorescence from chromophores excited through energy transfer (FRET) is one of the major techniques, and in this relatively mature field, many recent advances such as FRET imaging microscopy ${ }^{93,94}$ reflect an exploitation of technical developments. Burgeoning applications are to be found in the biophotonics area, in particular. ${ }^{95-97}$ Staying with molecular systems, the ongoing development of OLED emitters is also worth flagging as a prominent area of current activity. However, in connection with synthetic solid state systems where some of the more recently envisaged applications are being developed, one can see tangible signs of the emergence of another new research forefront.

One issue that would appear to merit much more consideration is the extent to which RET can be tailored, or even suppressed, in optical microcavity configurations. It is already well known that spontaneous emission is significantly influenced by inclusion of the donor in a cavity, through the restrictions that are thereby imposed on the sustainable optical modes. Barnes and others ${ }^{98-100}$ have drawn attention to the operation of similar principles in connection with donor-acceptor systems. In an impressive series of works by Lovett et al. ${ }^{62,63}$ (see also the references therein), several applications relating to quantum dot energy transfer processes have been considered, focusing in particular on the interplay of quantum state entanglement and Förster coupling. It has been suggested that these and other principles concerning the effect of static electric fields on coupled quantum dots may have an important role to play in quantum logic applications. Andrews has recently proposed a system in which the simultaneous delivery of laser pulses, with two differing off-resonant 
frequencies, might achieve a similar purpose, ${ }^{101,102}$ while other studies currently underway are beginning to explore novel nanomechanical effects produced by the action of RET. ${ }^{103}$

\subsection{Conclusion}

Resonance energy transfer is a subject that has come of age. Having been studied already for more than half a century, it has many established applications, despite the fact that some of the fundamental principles have only quite recently become fully understood. Quantum electrodynamics has played an important part in consolidating the theory, and has paved the way for a number of newer developments in which photonic and quantum optical aspects of light-matter coupling come into play. With such an extensive involvement in widely varying media, this is a subject that deserves to be more widely taught and understood; this chapter is offered as a contribution towards that objective.

\section{Acknowledgements}

It is a pleasure to acknowledge the involvement of several past and present members of my group in the work reported here, and I gladly acknowledge their invaluable contributions. In particular, I must thank Philip Allcock, David Bradshaw, Richard Crisp, Gareth Daniels, Robert Jenkins, Gediminas Juzeliūnas, and Justo Rodríguez. I also thank a number of these people, as well as Luciana Dávila Romero, and Jamie Leeder for producing most of the figures. I gratefully acknowledge funding support from the Engineering and Physical Sciences Research Council, the Leverhulme Trust, and the Royal Society.

\section{References}

1. V. M. Agranovich and M. D. Galanin, Electronic Excitation Energy Transfer in Condensed Matter, Elsevier/North-Holland, Amsterdam, The Netherlands, (1982).

2. B. W. van der Meer, J. Coker III, and S.-Y. S. Chen, Resonance Energy Transfer: Theory and Data, VCH, New York (1982).

3. D. L. Andrews and A. A. Demidov, Eds., Resonance Energy Transfer, Wiley, New York (1999).

4. G. W. Robinson and R. P. Frosch, "Electronic excitation transfer and relaxation,” J. Chem. Phys. 38, 1187-1203 (1963).

5. H. van Amerongen, L. Valkunas, and R. van Grondelle, Photosynthetic Excitons, World Scientific, Singapore (2000).

6. D. M. Guldi, "Fullerene-porphyrin architectures; photosynthetic antenna and reaction center models," Chem. Soc. Rev. 31, 22-36 (2002).

7. D. L. Dexter, "A theory of sensitized luminescence in solids," J. Chem. Phys. 21, 836-850 (1953). 
8. G. D. Scholes and K. P. Ghiggino, "Electronic interactions and interchromophore excitation transfer," J. Phys. Chem. 98, 4580-4590 (1994).

9. E. K. L. Yeow and K. P. Ghiggino, "Electronic energy transfer in multichromophoric arrays. The effects of disorder on superexchange coupling and energy transfer rate,” J. Phys. Chem. A 104, 5825-5836 (2000).

10. T. Förster, "Zwischenmolekulare Energiewanderung und Fluoreszenz," Annalen. Phys. 2, 55-75 (1948).

11. S. A. Latt, H. T. Cheung, and E. R. Blout, "Energy transfer: a system with relatively fixed donor-acceptor separation,” J. Amer. Chem. Soc. 87, 9951003 (1965).

12. J. R. Lakowicz, Principles of Fluorescence Spectroscopy, $2^{\text {nd }}$ edition, Chapter 10, Kluwer Academic, New York (1999).

13. B. Valeur, Molecular Fluorescence. Principles and Applications, Chapter 9, Wiley-VCH, Weinheim, Germany (2002).

14. C. Devadoss, P. Bharathi, and J. S. Moore, "Energy transfer in dendritic macromolecules: molecular size effects and the role of an energy gradient," J. Amer. Chem. Soc. 118, 9635-9644 (1996).

15. C. G. dos Remedios and P. D. J Moens, "Fluorescence resonance energy transfer spectroscopy is a reliable "ruler" for measuring structural changes in proteins: dispelling the problem of the unknown orientation factor," $J$. Struct. Biol. 115, 175-185 (1995).

16. L. Stryer and R. P. Haugland, Energy transfer: a spectroscopic ruler,” Proc. Natl. Acad. Sci. USA 58, 719-726 (1967).

17. G. D. Scholes and D. L. Andrews, "Damping and higher multipole effects in the quantum electrodynamical model for electronic energy transfer in the condensed phase,” J. Chem. Phys. 107, 5374-5384 (1997).

18. A. Salam, "A general formula for the rate of resonant transfer of energy between two electric multipole moments of arbitrary order using molecular quantum electrodynamics,” J. Chem. Phys. 122, 044112 (2005).

19. D. P. Craig and T. Thirunamachandran, Molecular Quantum ElectroDynamics, Dover, New York (1998).

20. R. G. Woolley, "Charged particles, gauge invariance, and molecular electrodynamics,” Int. J. Quantum. Chem. 74, 531-545 (1999).

21. R. G. Woolley, "Gauge invariance in non-relativistic electrodynamics," Proc. R. Soc. London, Ser. A 456, 1803-1819 (2000).

22. J. S. Avery, "Resonance energy transfer and spontaneous photon emission," Proc. Phys. Soc. 88, 1-8 (1966).

23. L. Gomberoff and E. A. Power, "The resonance transfer of excitation," Proc. Phys. Soc. 88, 281-284 (1966). 
24. D. L. Andrews, "A unified theory of radiative and radiationless molecular energy transfer,” J. Chem. Phys. 135, 195-201 (1989).

25. G. J. Daniels, R. D. Jenkins, D. S. Bradshaw, and D. L. Andrews, "Resonance energy transfer: The unified theory revisited," J. Chem. Phys. 119, 2264-2274 (2003).

26. D. L. Andrews and B. S. Sherborne, "Resonant excitation transfer: A quantum electrodynamical study,” J. Chem. Phys. 86, 401l-4017 (1987).

27. G. Juzeliūnas and D. L. Andrews, "Quantum electrodynamics of resonance energy transfer, Adv. Chem. Phys. 112, 357-410 (2000).

28. J. D. Jackson, Classical Electrodynamics, 3rd Edition, Wiley, New York, p. 411 (1999).

29. D. L. Andrews and D. S. Bradshaw, "Virtual photons, dipole fields and energy transfer: A quantum electrodynamical approach,” Eur. J. Phys. 25, 845-858 (2004).

30. F. Halzen and A. D. Martin, Quarks and Leptons, Wiley, New York, p. 140 (1984).

31. G. Juzeliūnas and D. L. Andrews, "Quantum electrodynamics of resonant energy transfer in condensed matter,” Phys. Rev. B 49, 8751-8763 (1994).

32. E. Fermi, Nuclear Physics, University of Chicago Press, Chicago (1950).

33. D. L. Andrews and G. Juzeliūnas, "Intermolecular energy transfer: retardation effects,” J. Chem. Phys. 96, 6606-6612 (1992).

34. R. D. Jenkins, G. J. Daniels, and D. L. Andrews, "Quantum pathways for resonance energy transfer,” J. Chem. Phys. 120, 11442-11448 (2004).

35. L. Novotny and B. Hecht, Principles of Nano-optics, University Press, Cambridge, UK, 285-293 (2006).

36. M. N. Berberan-Santos, E. J. N. Pereira, and J. M. G. Martinho, "Stochastic theory of combined radiative and nonradiative transport," J. Chem. Phys. 107, 10480-10484 (1997).

37. J. Baumann and M. D. Fayer, "Excitation transfer in disordered twodimensional and anisotropic three-dimensional systems: effects of spatial geometry on time-resolved observables,” J. Chem. Phys. 85, 4087-4107 (1986).

38. B. Nickel, "Orientation factor in Förster energy-transfer with photoselection of donor and acceptor," J. Chem. Phys. 198, 353-371 (1995).

39. B. W. van der Meer, in Resonance Energy Transfer, D. L. Andrews and A. A. Demidov, Eds., John Wiley and Sons, New York, 151-172 (1999).

40. D. L. Andrews and G. Juzeliūnas, "The range dependence of fluorescence anisotropy in molecular energy transfer,” J. Chem. Phys. 95, 5513-5518 (1991). 
41. V. V. Ovsyankin, in Spectroscopy of Solids Containing Rare Earth Ions, A. A. Kaplyanskii and R. M. Macfarlane, Eds., Elsevier, Amsterdam, 343-480 (1987).

42. C. Candu, V. Ciobu, and V. Enaki, "Four-center resonance energy transfer in rare earth doped crystals," J. Optoelectron. Adv. Mater. 7, 781-786 (2005).

43. D. L. Dexter, "Possibility of luminescent quantum yields greater than unity,” Phys. Rev. 108, 630-633 (1957).

44. N. Bloembergen, "Solid state infrared quantum counters," Phys. Rev. Lett. 2, 84-85 (1959).

45. T. Miyakawa and D. L. Dexter, "Cooperative and stepwise excitation of luminescence: trivalent rare-earth ions in $\mathrm{Yb}^{3+}$-sensitized crystals,” Phys. Rev. B 1, 70-80 (1970).

46. F. Auzel, "Upconversion processes in coupled ion systems," J. Lumin. 45, 341-345 (1990).

47. F. Auzel, “Compteur quantique par transfert d'energie entre deux ions de terre rares dans un tungstate mixte et dans un verre," Compt. Rend. 262B, 1016-1021 (1966).

48. M. Chua and P. A. Tanner, "Three-body energy transfer processes of lanthanide ions in crystals,” J. Lumin. 66-7, 203-207 (1995).

49. R. D. Jenkins, D. L. Andrews, and L. C. Dávila Romero, "A new diagrammatic methodology for non-relativistic quantum electrodynamics," J. Phys. B: Atom. Molec. Opt. Phys. 35, 445-468 (2002).

50. R. D. Jenkins and D. L. Andrews, "Three-center systems for energy pooling: quantum electrodynamical theory,” J. Phys. Chem. A 102, 1083410842 (1998).

51. D. L. Andrews, "An accretive mechanism for blue-shifted fluorescence in strongly-pumped systems: resonance energy transfer with Raman emission," J. Raman Spectrosc. 31, 791-796 (2000).

52. D. L. Andrews and R. D. Jenkins, "A quantum electrodynamical theory of three-center energy transfer for upconversion and downconversion in rare earth doped materials," J. Chem. Phys. 114, 1089-1100 (2001).

53. X. Zhang, J.-P. Jouart, and G. Mary, "Energy transfer upconversion in $\mathrm{Ho}^{3+}$ and $\mathrm{Ho}^{3+}, \mathrm{Yb}^{3+}$ doped $\mathrm{CdF}_{2}$ crystals,” J. Phys. Condens. Matter 10, 493-500 (1998).

54. R. T. Wegh, H. Donker, A. Meijerink, R. J. Lamminmăki, and J. Hölsä, "Vacuum-ultraviolet spectroscopy and quantum cutting for $\mathrm{Gd}^{3+}$ in $\mathrm{LiYF}_{4}$," Phys. Rev. B 56, 13841-13488 (1997).

55. C. R. Kagan, C. B. Murray, M. Nirmal, and M. G. Bawendi, "Electronic energy transfer in CdSe quantum dot solids,” Phys. Rev. Lett. 76, 15171520 (1997). 
56. S. A. Crooker, J. A. Hollingsworth, S. Tretiak, and V. I. Klimov, "Spectrally resolved dynamics of energy transfer in quantum-dot assemblies: Towards engineered energy flows in artificial materials,” Phys. Rev. Lett. 89, 186802 (2002).

57. B. W. Lovett, J. H. Reina, A. Nazir, B. Kothari, and G. A. Briggs, "Resonant transfer of excitons and quantum computation," Phys. Lett. A 315, 136-142 (2003).

58. A. O. Govorov, "Spin and energy transfer in nanocrystals without tunneling,” Phys. Rev. B 68, 075315 (2003).

59. H. Kamada and H. Gotoh, "Quantum computation with quantum dot excitons,” Semiconductor Sci. Tech. 19, S392-S396 (2004).

60. S. Sangu, K. Kobayashi, A. Shojiguchi, and M. Ohtsu, "Logic and functional operations using a near-field optically coupled quantum-dot system,” Phys. Rev. B 69, 115334 (2004).

61. A. R. Clapp, I. L. Medintz, H. T. Uyeda, B. R. Fisher, E. R. Goldman, M. G. Bawendi, and H. Mattoussi, "Quantum dot-based multiplexed fluorescence resonance energy transfer,” J. Amer. Chem. Soc. 127, 18212-18221 (2005).

62. B. W. Lovett, J. H. Reina A. Nazir, and G. A. D. Briggs, "Optical schemes for quantum computation in quantum dot molecules," Phys. Rev. B 68, 205319 (2003).

63. A. Nazir, B. W. Lovett, S. D. Barrett, J. H. Reina, and G. A. D. Briggs, “Anticrossings in Förster coupled quantum dots," Phys. Rev. B 71, 045334 (2005).

64. L. Bányai and S.W. Koch, Semiconductor Quantum Dots, World Scientific, Singapore (1993).

65. J. M. Villas-Boas, S. E. Ulloa, and A. O. Govorov, "Photocurrent and spin manipulation in quantum dots,” Physica E: Low-dimensional Systems and Nanostructures 34, 333-335 (2006).

66. G. D. Scholes and D. L. Andrews, "Resonance energy transfer and quantum dots,” Phys. Rev. B 72, 125331 (2005).

67. G. D. Scholes, R. D. Harcourt, and G. R. Fleming, "Electronic interactions in photosynthetic light-harvesting complexes: The role of carotenoids," $J$. Phys. Chem. B $1017302-7312$ (1997).

68. B. P. Krueger, G. D. Scholes, R. Jimenez, and G. R. Fleming, "Electronic excitation transfer from carotenoid to bacteriochlorophyll in the purple bacterium Rhodopseudomonas acidophila,” J. Phys. Chem. B 102, 22842292 (1998).

69. S. Gnanakaran, R. Haran, R. Kumble, and R. M. Hochstrasser, "Energy transfer and localization: applications to photosynthetic systems," in Resonance Energy Transfer, D. L. Andrews and A. A. Demidov, Eds., Wiley, New York, 308-365 (1999). 
70. R. van Grondelle and O. J. G. Samsen, in Resonance Energy Transfer, D. L. Andrews and A. A. Demidov, Eds., Wiley, New York, 366-398 (1999).

71. S. Savikhin, D. R. Buck, and W. S. Struve, in Resonance Energy Transfer, D. L. Andrews and A. A. Demidov, Eds., Wiley, New York, 399-434 (1999).

72. G. S. Engel, T. R. Calhoun, E. L. Read, T.-K. Ahn, T. Mancal, Y.-C. Cheng, R. E. Blankenship, and G. R. Fleming, "Evidence for wavelike energy transfer through quantum coherence in photosynthetic systems," Nature 446, 782-786 (2007).

73. X. Hu and K. Schulten, "How Nature harvests sunlight,” Phys. Today $\mathbf{5 0}$ (8), 28-34 (1997).

74. T. Renger, V. May, and O. Kühn, "Ultrafast excitation energy transfer dynamics in photosynthetic pigment-protein complexes,” Phys. Rep. 343, 138-254 (2001)

75. T. Brixner, J. Stenger, H. M. Vaswani, M. Cho, R. E. Blankenship, and G. R. Fleming, "Two-dimensional spectroscopy of electronic couplings in photosynthesis," Nature 434, 625-628 (2005).

76. A. Adronov and J. M. J. Fréchet, "Light-harvesting dendrimers," Chem. Commun.1701-1710 (2000).

77. P. Ball, "Natural strategies for the molecular engineer," Nanotechnology 13, R15-R28 (2002).

78. D. L. Andrews, "Optical energy harvesting materials," in Introduction to Complex Mediums for Optics and Electromagnetics, W. S. Weiglhofer and A. Lakhtakia, Eds., SPIE Press, Bellingham, WA, 141-163 (2003).

79. D. L. Andrews, Ed., Energy Harvesting Materials, World Scientific, Singapore (2005).

80. A. Bar-Haim, J. Klafter, and R. Kopelmann, "Dendrimers as controlled artificial energy antennae,” J. Amer. Chem. Soc. 119, 6197-6198 (1997).

81. A. Nantalaksakul, D. R. Reddy, C. J. Bardeen, and S. Thayumanavan, "Light harvesting dendrimers," Photosynthesis Res. 87, 133-150 (2006).

82. C. Devadoss, P. Bharathi, and J. S. Moore, "Energy transfer in dendritic macromolecules: Molecular size effects and the role of an energy gradient," J. Amer. Chem. Soc. 118, 9635-9644 (1996).

83. S. F. Swallen, Z.-Y. Shi, W. Tan, Z. Xu, J. S. Moore, and R. Kopelman, "Exciton localisation hierarchy and directed energy transfer in conjugated linear aromatic chains and dendrimeric supermolecules,” J. Lumin. 76-7, 193-196 (1998).

84. A. Archut and F. Vögtle, "Functional cascade molecules," Chem. Soc. Rev. 27, 233-240 (1998). 
85. P. G. van Patten, A. P. Shreve, J. S. Lindsey, and R. J. Donohoe, "Energytransfer modeling for the rational design of multiporphyrin light-harvesting arrays,” J. Phys. Chem. B 102, 4209-4216 (1998).

86. D. L. Andrews and R. G. Crisp, "Theory of directed electronic energy transfer,” J. Fluorescence 16, 191-199 (2006).

87. D. L. Andrews and J. Rodríguez, "Resonance energy transfer: Spectral overlap, efficiency and direction,” J. Chem. Phys. 127, 084509 (2007).

88. D. L. Andrews and A. M. Bittner, "Energy transfer in a static electric field," J. Lumin. 55, 231-242 (1993).

89. P. Allcock, R. D. Jenkins, and D. L. Andrews, "Laser-assisted resonanceenergy transfer, Phys. Rev. A 61, 023812 (2000).

90. D. L. Andrews and R. G. Crisp, "Optically activated energy transfer: array implementation,” J. Opt. A: Pure Appl. Opt. 8, S106-S112 (2006).

91. B. W. Alligood and A. Salam, "On the application of state sequence diagrams to the calculation of the Casimir-Polder potential," Mol. Phys. 105, 395-404 (2007).

92. D. S. Bradshaw and D. L. Andrews, "Optically controlled resonance energy transfer: Mechanism and configuration for all-optical switching," J. Chem. Phys. 128, 144506 (2008).

93. M. Elangovan, H. Wallrabe, Y. Chen, R. N. Day, M. Barroso, and A. Periasamy, "Characterization of one- and two-photon excitation fluorescence resonance energy transfer microscopy,” Methods 29, 58-73 (2003).

94. S. K. Sekatskii, "Fluorescence resonance energy transfer scanning near-field optical microscopy, Phil. Trans. R. Soc. A: Math. Phys. Eng. Sci. 362, 901919 (2004).

95. R. MacColl, Allophycocyanin and energy transfer, Biochim. Biophys. Acta Bioenergetics 1657, 73-81 (2004).

96. G. Ramanoudjame, M. Du, K. A. Mankiewicz, and V. Jayaraman, "Allosteric mechanism in AMPA receptors: A FRET-based investigation of conformational changes,” Proc. Natl. Acad. Sci. USA 103, 10473-10478 (2006).

97. X. You, A. W. Nguyen, A. Jabaiah, M. A. Sheff, K. S. Thorn, and P. S. Daugherty, "Intracellular protein interaction mapping with FRET hybrids," Proc. Natl. Acad. Sci. USA 103, 18458-18463 (2006).

98. M. Cho, "Confinement-induced enhancement or suppression of the resonant dipole-dipole interaction,” J. Chem. Phys. 110, 4998-5010 (1999).

99. P. Andrew and W. L. Barnes, "Förster energy transfer in an optical microcavity," Science 290, 785-788 (2000).

100. W. L. Barnes, "Spontaneous emission and energy transfer in the optical microcavity," Contemp. Phys. 41, 287-300 (2000). 
101. D. L. Andrews, "Optically switched energy transfer: Twin-beam offresonance control,” Phys. Rev. Lett. 99, 023601 (2007).

102. D. L. Andrews, R. G. Crisp, and S. Li, "Single and dual beam optical switching of resonance energy transfer," J. Chem. Phys. 127, 174702 (2007).

103. D. L. Andrews, D. S. Bradshaw, J. Leeder, and J. Rodríguez, "Dynamics of the dispersion interaction in an energy transfer system," Phys. Chem. Chem. Phys. 10, 5250-5255 (2008).

\section{Biography}

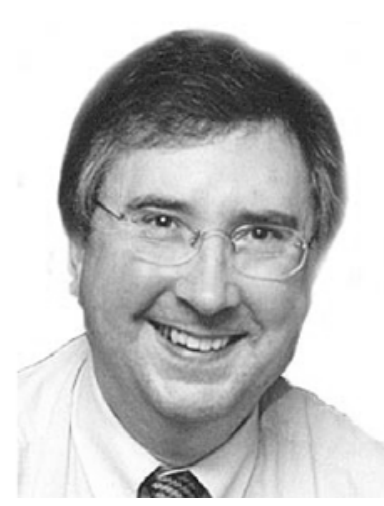

David L. Andrews followed a degree in Chemistry at University College London with doctoral studies, then a postdoctoral appointment in Mathematics, before moving to a lectureship at University of East Anglia (UEA) in Norwich, U.K. He gained a Chair in Chemical Physics in 1996. Andrews was elected a Fellow of the Royal Society of Chemistry in 1988, a Fellow of the Institute of Physics in 1999, and a Fellow of SPIE, the International Society for Optical Engineering, in 2006. His research centres on molecular photophysics, utilising quantum electrodynamics to develop theory for energy transport and a range of multiphoton and other nonlinear optical phenomena. Andrews's early work led towards the unified theory of energy transfer accommodating both radiationless and radiative mechanisms. His group was the first to identify and predict the characteristics of two-photon resonance energy transfer, anticipating later experiments on biological systems. Currently his group's studies focus on nonlinear optical processes, optical binding, and energy harvesting in nanosystems. Andrews has over 200 papers and ten books to his name, including the textbook Lasers in Chemistry, Springer Publishing Co. 